\title{
Neutron star radii and crusts: Uncertainties and unified equations of state
}

\author{
M. Fortin, ${ }^{1,{ }^{*}}$ C. Providência, ${ }^{2}$ Ad. R. Raduta, ${ }^{3}$ F. Gulminelli, ${ }^{4}$ J. L. Zdunik, ${ }^{1}$ P. Haensel, ${ }^{1}$ and M. Bejger ${ }^{1}$ \\ ${ }^{1}$ N. Copernicus Astronomical Center, Polish Academy of Sciences, Bartycka 18, 00-716 Warszawa, Poland \\ ${ }^{2}$ CFisUC, Department of Physics, University of Coimbra, P-3004-516 Coimbra, Portugal \\ ${ }^{3}$ IFIN-HH, P.O. Box MG6, Bucharest-Magurele, Romania \\ ${ }^{4}$ Université de Caen Normandie, ENSICAEN, UNICAEN, CNRS/IN2P3, LPC Caen, 14000 Caen, France
}

(Received 6 April 2016; revised manuscript received 12 July 2016; published 19 September 2016)

\begin{abstract}
The uncertainties in neutron star radii and crust properties due to our limited knowledge of the equation of state are quantitatively analyzed. We first demonstrate the importance of a unified microscopic description for the different baryonic densities of the star. If the pressure functional is obtained matching a crust and a core equation of state based on models with different properties at nuclear matter saturation, the uncertainties can be as large as $\sim 30 \%$ for the crust thickness and $4 \%$ for the radius. Necessary conditions for causal and thermodynamically consistent matchings between the core and the crust are formulated and their consequences examined. A large set of unified equations of state for purely nucleonic matter is obtained based on twenty-four Skyrme interactions and nine relativistic mean-field nuclear parametrizations. In addition, for relativistic models fifteen equations of state including a transition to hyperonic matter at high density are presented. All these equations of state have in common the property of describing a $2 M_{\odot}$ star and of being causal within stable neutron stars. Spans of $\sim 3$ and $\sim 4$ $\mathrm{km}$ are obtained for the radius of, respectively, $1.0 M_{\odot}$ and 2.0 $M_{\odot}$ stars. Applying a set of nine further constraints from experiment and $a b$ initio calculations the uncertainty is reduced to $\sim 1$ and $2 \mathrm{~km}$, respectively. These residual uncertainties reflect lack of constraints at large densities and insufficient information on the density dependence of the equation of state near the nuclear matter saturation point. The most important parameter to be constrained is shown to be the symmetry energy slope $L$. Indeed, this parameter exhibits a linear correlation with the stellar radius, which is particularly clear for small mass stars around $1.0 M_{\odot}$. The other equation-of-state parameters do not show clear correlations with the radius, within the present uncertainties. Potential constraints on $L$, the neutron star radius, and the equation of state from observations of thermal states of neutron stars are also discussed. The unified equations of state are made available in the Supplemental Materials and via the CompOSE database.
\end{abstract}

DOI: 10.1103/PhysRevC.94.035804

\section{INTRODUCTION}

Simultaneous measurements of the masses and radii of neutron stars (NS), if sufficiently precise, will impose strong constraints on the equation of state (EOS) of dense matter significantly above (standard) nuclear (baryon number) density $n_{0}=0.16 \mathrm{fm}^{-3}$. The value of $n_{0}$ is a suitable unit to measure the baryon (number) density in NS cores. In fact, the two most massive pulsars PSR J0348+0432 and PSR J1614-2230 alone, with a mass close to $2 M_{\odot}[1-3]$, already put quite stringent constraints on the EOS in the $5 n_{0}-8 n_{0}$ density range. These mass measurements are particularly relevant to assess the possible existence of exotic phases of dense matter in the cores of massive NS.

Significant effort has been put into the determination of the radii of NS, but presently there is still a large uncertainty associated with this quantity; see the discussion in [4-6]. Particularly interesting is the measurement of radii for the stellar mass range $1.3 M_{\odot}-1.5 M_{\odot}$, where on the one hand many precise NS mass measurements exist, and on the other hand dense matter theories predict a nearly constant value of $R$ (albeit different for various dense matter theories). We expect that up to $2 n_{0}-3 n_{0}$ NS matter involves nucleons only and therefore that the radius for the "canonical" NS mass $1.4 M_{\odot}$, denoted usually as $R_{1.4}$, characterizes the EOS in

*Corresponding author: fortin@camk.edu.pl the nucleon segment. Recently, a new constraint has been added to this discussion. According to Ref. [7], an EOS with $M_{\text {max }}>2 M_{\odot}$ should produce $R_{1.4} \gtrsim 10.7 \mathrm{~km}$ in order to avoid being noncausal at highest NS densities.

We expect that future simultaneous determinations of the mass and radius of a NS with a $5 \%$ precision will be possible through the analysis of the x-ray emission of NS, thanks to the forthcoming NICER [8], Athena [9] and LOFT-like [10] missions. It is therefore important to be able to quantify the uncertainties introduced in the NS mass and radius calculations simultaneously by the approximations used when constructing the complete EOS for stellar matter, by the scarcely available constraints on the EOS at high densities and large isospin asymmetries, and by the lack of information about the possible exotic states of the matter existing in the interior of a NS.

In the present work we aim to understand how the calculation of the NS radii are affected by the EOS of the crust, having in mind that the EOS constructed to describe NS matter are typically non-unified, i.e., built piecewise starting from different models for each sector of NS matter. This is to be contrasted with unified EOS, where all segments (outer crust, inner crust, liquid core) are calculated starting from the same nuclear interaction. In practice, for a NS crust with $n \lesssim$ $10^{-4} n_{0}$ one uses experimental nuclear masses. For higher crust densities, where the relevant experimental nuclear masses are not available, they should be calculated theoretically. Usually, one employs an effective nuclear Hamiltonian (or Lagrangian) 
and a many-body method that makes the calculation feasible (e.g., the Thomas-Fermi approximation or the compressible liquid-drop model). It should be mentioned that some minor matching problems exist already at the transition between the experimentally based low-density segment of the EOS and that obtained with an effective nuclear interaction, if the latter does not fit perfectly experimental nuclear masses. However, examples in the present paper show that resulting uncertainty in $R$ is very small. The calculated EOS for the crust will depend on the assumed effective nuclear interaction, but the phase transition between the inner crust (including a possibility of a bottom layer with nuclear pastas) and the liquid core will be described correctly. The EOS is then continuous through the whole NS core, and yields a unique $R(M)$ for each effective interaction, with negligible residual model dependence.

In contrast, in the standard case of a non-unified EOS model, the resulting $R(M)$ depends on the procedure of matching the crust and core EOS segments. As an example, in [11] it is proposed that the Baym-Pethick-Sutherland (BPS) EOS [12] be chosen to describe the crust and a matching of the crust EOS to the core one is performed at $0.01 \mathrm{fm}^{-3}$, while the core is described within a relativistic mean field (RMF) approach allowing for fitting several parameters of nuclear matter at saturation. Similarly a parametrization of the high-density equation of state based on piecewise polytropes is presented in [13] and allows one to systematically study the effect of observational constraints on the EOS of cold stellar matter. Although for the high density range several models have been considered, for low densities a single EOS, the one of Douchin and Haensel [14] based on a specific Skyrme interaction, namely SLy4 [15], is used. In an equivalent way, the authors of [5] have studied constraints on the NS structure by considering two classes of EOS models, and in both the BPS EOS was taken for the low density EOS, alone or supplemented by the Negele-Vautherin EOS [16]. Both of these models are based on old energy functionals which do not fulfill present experimental nuclear physics constraints. In all these examples, one can wonder by how much the simplified choice for the subnuclear density EOS affects the conclusions obtained from experimental and observational constraints on the EOS. In fact, in [17] it has been argued that, depending on the assumed properties of the low density EOS, it is possible to obtain pressures at the crust-core transition large enough to explain the large Vela glitches, even considering the entrainment effect. This indicates that a proper description of the crust and the crust-core transition as well as a sensitivity study and a systematic uncertainty evaluation are required.

In the present paper, we will first study how the matching of the crust EOS with the core one affects the NS radius and the crust thickness, when models that describe the crust and the core EOS are not the same. In order to reduce the uncertainties introduced on the calculation of the star structure, some general indications will be presented on how to build a non-unified EOS.

Next we will take a set of unified EOS obtained in the framework of the RMF models and Skyrme interactions. For both frameworks we restrict ourselves to EOS that are able to describe a $2 M_{\odot}$ star and remain causal; a nontrivial condition for the second set of nonrelativistic models.
In the case of the RMF models one can consider also their extensions allowing for the presence of hyperons. Vectormeson couplings to hyperons are obtained assuming the SU(6) symmetry. Repulsion in the hyperon sector associated with their coupling to a hidden-strangeness vector-isoscalar meson $\phi$ allows for $M>2 M_{\odot}$. We also study how adding the hiddenstrangeness scalar-isoscalar meson $\sigma^{\star}$ to get a weak $\Lambda \Lambda$ attraction softens the EOS. In principle the same exercise could be done for the nonrelativistic models. However, the present uncertainties in the hyperon-nucleon and hyperon-hyperon interactions are such that the introduction of hyperon degrees of freedom is still extremely model dependent. In particular, the most sophisticated many-body approaches available in the literature [18] either did not yet succeeded in producing $2 M_{\odot}$ stars, or cannot deal with the full baryonic octet [19]. However even in the case of RMF, strong uncertainties are associated to the couplings. We make all the EOS used here available in the Supplemental Material and via the CompOSE database [20].

Within our large set of unified EOS we will study the dependence of the NS radius and the thickness of the crust on the mass in order to pin down the residual uncertainties due to our imperfect knowledge of the EOS parameters. As we remind in Sec. II, the EOS of nuclear matter near $n_{0}$ and for small neutron excess is constrained by the semiempirical evaluations of nuclear matter parameters extracted from nuclear physics data. We will seek the correlations between theoretically calculated nuclear matter parameters near $n_{0}$ and NS structure. We will specifically show that the best correlation is obtained between the radius of light NS with $M \leqslant 1.4 M_{\odot}$ and the symmetry energy slope $L$. This confirms that indeed the $L$ parameter is the most important one to be constrained from laboratory experiments and/or $a b$ initio calculations. A most crucial constraint could potentially come from the threshold density above which the direct Urca (DUrca) process operates. Indeed the interval of $L$ which is compatible with terrestrial constraints largely overlaps with the one for which the nucleonic DUrca process does not operate in massive NS. In turn, the presence of nucleonic DUrca appears to be needed in order to explain the thermal states of accreting neutron stars [21]. This means that combining radii measurements with observations of thermal states of NS might constitute a very stringent test for the EOS.

The plan of the paper is as follows. In Sec. II we give a very general overview of nuclear matter in NS. We also establish notations for nuclear matter and its relation to the semi-empirical nuclear-matter parameters. Section III describes the different techniques that are used to match the crust and core EOS, and the resulting uncertainty associated with the star radius and the crust thickness. The relativistic and nonrelativistic unified EOS employed for this work are described in Sec. IV, and the corresponding $M(R)$ relations are given. Section V contains the main results of this work. The predictions for the radius and crust thickness are given, the correlation between the radius and the EOS parameters is discussed, and the different unified EOS are compared to the terrestrial constraints. Potential constraints from the necessity of DUrca processes to explain low-luminosity NS are presented. Finally Sec. VI concludes the paper. 


\section{NUCLEAR MATTER IN NEUTRON STARS AND SEMI-EMPIRICAL PARAMETERS}

Consider the NS interior from the very basic point of view of nuclear matter states relevant for each main NS layer. The $T=0$ approximation can be used since the Fermi energy of the nucleons is much larger than the thermal energy associated with the temperatures of $\sim 10^{7}-10^{9} \mathrm{~K}$ expected inside NS. The outer core consists of a lattice of nuclear-matter droplets permeated by an electron gas. The inner crust is made of a lattice of nuclear-matter droplets coexisting with a neutron gas. With increasing pressure, droplets can become unstable with respect to merging into infinite nuclear matter structures (rods, plates) immersed in a neutron gas. The plates of nuclear matter then glue together leaving tubes filled with neutron gas, then the tubes break into bubbles of neutron gas in nuclear matter. Both the inner crust and the (possible) mantle of nuclear pastas form inhomogeneous two-phase states of nucleon matter.

At the edge of the outer core, inhomogeneous nucleon matter coexists with uniform homogeneous nuclear matter. To model it, we consider a mixture of strongly interacting neutrons and protons, with Coulomb interactions switched off. Let us define the baryon number density $n=n_{n}+n_{p}$ and the neutron excess parameter $\delta=\left(n_{n}-n_{p}\right) / n$. The energy per nucleon (excluding the nucleon rest energy) is $E_{\mathrm{NM}}(n, \delta)$. Theoretical models of nuclear matter give $E_{\mathrm{NM}}(n, \delta)$ and yield a set of parameters that characterize the EOS near the saturation point (minimum of $E_{\mathrm{NM}}$ ) and for small $\delta$. For a given model, the minimum of energy per nucleon, $E_{\mathrm{s}}$, is reached at the saturation density $n=n_{\mathrm{s}}$ and for $\delta=0$.

The difference between the calculated values for the saturation density $n_{\mathrm{s}}$ and the commonly used normal nuclear density $n_{0}$ defined in the first sentence of Sec. I deserves a comment. The values of $n_{\mathrm{s}}$ are model dependent and vary between 0.146 and $0.154 \mathrm{fm}^{-3}$ for the RMF models (Table II) and between 0.151 and $0.165 \mathrm{fm}^{-3}$ for the Skyrme models (Table IV). The use of a precise value of $n_{\mathrm{s}}$ is crucial for the correct calculation of the EOS. In contrast, $n_{0}$ is just a chosen baryon number density unit.

Let us define the so-called symmetry energy,

$$
E_{\mathrm{sym}}(n)=\frac{1}{2}\left(\frac{\partial^{2} E_{\mathrm{NM}}}{\partial \delta^{2}}\right)_{\delta=0},
$$

and its value at saturation,

$$
J=E_{\mathrm{sym}}\left(n_{\mathrm{s}}\right)
$$

Two additional parameters related to the first and second derivatives of the symmetry energy at the saturation point are, respectively, the symmetry-energy slope parameter $L$,

$$
L=3 n_{\mathrm{s}}\left(\frac{d E_{\mathrm{sym}}}{d n}\right)_{n_{\mathrm{s}}},
$$

and the symmetry incompressibility $K_{\text {sym }}$,

$$
K_{\mathrm{sym}}=9 n_{\mathrm{s}}^{2}\left(\frac{d^{2} E_{\mathrm{sym}}}{d^{2} n}\right)_{n_{\mathrm{s}}} .
$$

Finally, the incompressibility at saturation $K$ is

$$
K=9 n_{\mathrm{s}}^{2}\left(\frac{\partial^{2} E_{\mathrm{NM}}}{\partial n^{2}}\right)_{n_{\mathrm{s}}, \delta=0} .
$$

Knowledge of parameters $\left\{n_{\mathrm{s}}, E_{\mathrm{s}}, K, J, \ldots,\right\}$ is sufficient to reproduce theoretical EOS of nuclear matter near the saturation point, a situation characteristic of laboratory nuclei. However, after being fine-tuned at the saturation point, the energy-density functionals are actually extrapolated up to $n \sim$ $8 n_{\mathrm{s}} \simeq 8 n_{0}$ and $\delta \simeq 1$, characteristic of the cores of massive NS. Therefore, making $\left\{n_{\mathrm{s}}, E_{\mathrm{s}}, K, J, \ldots,\right\}$ consistent with the semi-empirical evaluations of these parameters obtained, using a wealth of experimental data on atomic nuclei, yields constraints on the corresponding EOS of NS, and consequently, NS models, and in particular NS radii.

\section{NON-UNIFIED EQUATIONS OF STATE AND CORE-CRUST MATCHING}

In the present section we will discuss the problem of the core-crust matching of the EOS when a non-unified EOS is used to describe stellar matter. The use of a non-unified EOS will be shown not to affect the determination of the NS mass but to have a significant influence on the radius calculation.

\section{A. Different procedures for core-crust matching}

The determination of the mass and radius of a NS is possible from the integration of the Tolman-OppenheimerVolkoff (TOV) equations for spherical and static relativistic stars [22], given the EOS of stellar matter $P(\rho)$, where $P$ is the pressure and $\rho$ the energy density. The EOS for the whole NS is generally obtained by the matching of three different segments: the first one for the outer crust, the second one for the inner crust, and the last one for the core. The EOS for the outer crust, which extends from the surface to the neutron drip density, requires knowledge of the masses of neutron-rich nuclei [12,23-25]. This information comes from experiments or, when no information exists, from some energy-density functional calculations. The inner crust corresponds to a nonhomogeneous region between the neutron drip and the crust-core transition. This region may include nonspherical nuclear clusters, generally known as pasta phases [26], and has been described within several approaches [27-34]. Finally the core formed by a homogeneous liquid composed of neutrons, protons, electrons, muons, and possibly exotic matter, in $\beta$ equilibrium, extends from the crust-core transition to the center of the star. It should be pointed out, however, that in addition to exotic phases which can possibly appear at high densities, matter may also be nonhomogeneous in the core, e.g., in the form of a mixed hadron-quark phase [35]. In the present work we consider a homogeneous core.

Since the core accounts for most of the mass and radius of the star, authors frequently work with a non-unified EOS, and match the core EOS to one for the crust, in particular the Baym-Pethick-Sutherland (BPS) [12] together with the BaymBethe-Pethick (BBP) [36], the Negele-Vautherin (NV) [16], or the Douchin-Haensel (DH) [14]. The matching is generally done so that the pressure is an increasing function of the energy 

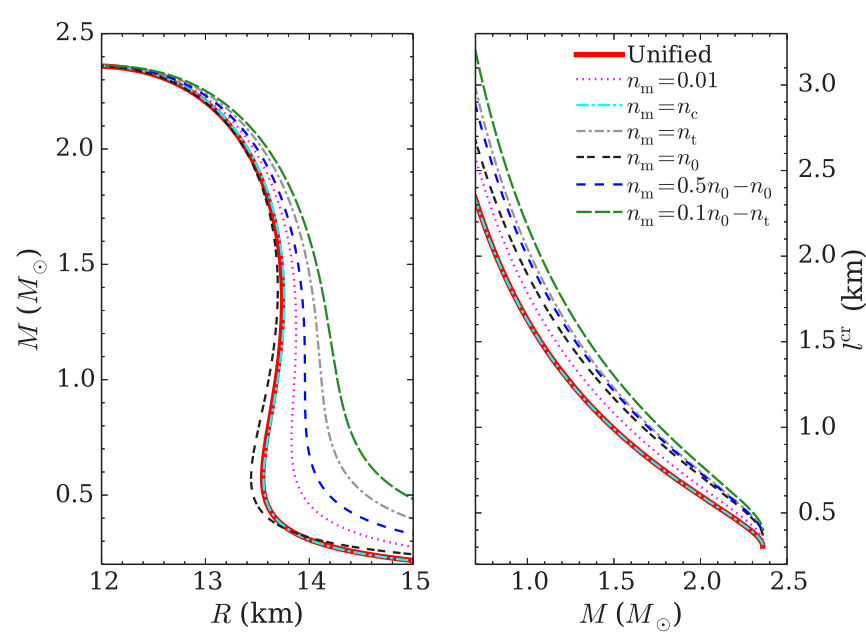

FIG. 1. Mass versus radius (left) and crust thickness $l^{\text {cr }}$ versus mass (right) for the relativistic mean field model GM1, using different matching procedures (see text).

density. This condition still leaves a quite large freedom in the matching procedure. In principle the matching procedures done at a specific density should be performed using a Maxwell construction, i.e., at constant baryonic chemical potential, so that the pressure is an increasing function of both the density and the chemical potential.

In the following a non-unified EOS is built from two different EOS. The one for the crust defined by $P_{\mathrm{cr}}, \rho_{\mathrm{cr}}, n_{\mathrm{cr}}$ is used up to $P_{1}, \rho_{1}, n_{1}$, while another one for the core, $P_{\mathrm{co}}, \rho_{\mathrm{co}}$, $n_{\mathrm{co}}$, is considered above $P_{2}, \rho_{2}, n_{2}$. The matching is performed in the region of pressure $P_{1} \leqslant P \leqslant P_{2}$, and if $P_{1} \neq P_{2}$ a linear interpolation between $\left(P_{1}, \rho_{1}\right)$ and $\left(P_{2}, \rho_{2}\right)$ is considered. The pressures $P_{1}$ and $P_{2}$ are generally defined at a reference density such as the neutron drip density $n_{\mathrm{d}}$, the crust-core transition density $n_{\mathrm{t}}$, the saturation density $n_{0}$, and the density $n_{\mathrm{c}}$ where the two EOS cross.

In Fig. 1 we plot the radius-mass curves (left) and the crust thickness (right) versus the star mass obtained with the GM1 parametrization [37] with a purely nucleonic core obtained for different gluing procedures:

(1) Unified: by unified we mean an EOS built with the DH EOS for the outer crust $\left(n \leqslant 0.002 \mathrm{fm}^{-3}\right)$ and the inner crust and core obtained within the same model, here GM1. The inner crust was calculated within a Thomas-Fermi calculation of the pasta phase [38] and the core EOS matches the inner crust at the crust-core transition density $n_{\mathrm{t}}$;

(2) $n_{1}=0.01 \mathrm{fm}^{-3}$ : the crust BPS+BBP EOS is glued to the core EOS at $0.01 \mathrm{fm}^{-3}$ as indicated in [11];

(3) $n_{1}=n_{\mathrm{c}}$ : the gluing is done at the density where the DH EOS and the core EOS cross as in [13];

(4) $n_{1}=n_{\mathrm{t}}$ : the DH EOS is considered for the crust and homogeneous matter EOS for $n>n_{\mathrm{t}}$.

(5) $n_{1}=n_{0}$ : the DH EOS is used for $n<n_{0}$ and the core EOS above the saturation density $n_{0}$.

(6) $n_{1}=0.5 n_{0}, n_{2}=n_{0}$ : DH EOS is used for $n<0.5 n_{0}$, the homogeneous matter EOS is used above $n_{0}$.
TABLE I. NS radii $R_{1}$ and $R_{1.4}$ (in $\mathrm{km}$ ) and crust thicknesses $l_{1}^{\text {cr }}$ and $l_{1.4}^{\text {cr }}$ (in $\mathrm{km}$ ) for masses of $1.0 M_{\odot}$ and $1.4 M_{\odot}$ for different matchings between the core and the crust. $\Delta x$ (in \%) for a given quantity $x$ corresponds to the relative difference between the value of $x$ for unified EOS and the one for a given matching. Three functionals are considered: NL3, NL3 $\omega \rho$, and GM1.

\begin{tabular}{|c|c|c|c|c|c|c|c|c|}
\hline & $R_{1}$ & $\Delta R_{1}$ & $R_{1.4}$ & $\Delta R_{1.4}$ & $l_{1}^{\mathrm{cr}}$ & $\Delta l_{1}^{\mathrm{cr}}$ & $l_{1.4}^{\mathrm{cr}}$ & $\Delta l_{1.4}^{\mathrm{cr}}$ \\
\hline \multicolumn{9}{|l|}{$\overline{\mathrm{GM} 1}$} \\
\hline nified & 13.71 & & 13.76 & & 1.62 & & 1.09 & \\
\hline$n=0.01$ & 13.86 & 1.09 & 13.86 & 0.73 & 1.78 & 9.88 & 1.19 & 9.17 \\
\hline$n_{\mathrm{t}}$ & 14.12 & 2.99 & 13.92 & 1.16 & 1.64 & 1.23 & 1.10 & 0.92 \\
\hline$n_{0}$ & 13.61 & -0.73 & 13.70 & -0.44 & 2.04 & 25.93 & 1.36 & 24.77 \\
\hline $0.5 n_{0}-n_{0}$ & 13.96 & 1.82 & 13.92 & 1.16 & 2.00 & 23.46 & 1.33 & 22.02 \\
\hline $0.1 n_{0}-n_{\mathrm{t}}$ & 14.27 & 4.08 & 14.12 & 2.62 & 2.18 & 34.57 & 1.44 & 32.11 \\
\hline Max. diff. & 0.66 & & 0.42 & & 0.56 & & 0.35 & \\
\hline \multicolumn{9}{|l|}{ NL3 } \\
\hline unified & 14.54 & & 14.63 & & 1.91 & & 1.30 & \\
\hline$n=0.01$ & 14.78 & 1.65 & 14.78 & 1.03 & 2.15 & 12.57 & 1.45 & 11.54 \\
\hline$n_{\mathrm{c}}$ & 14.97 & 2.96 & 14.91 & 1.91 & 2.35 & 23.04 & 1.58 & 21.54 \\
\hline$n_{\mathrm{t}}$ & 14.96 & 2.89 & 14.90 & 1.85 & 2.34 & 22.51 & 1.57 & 20.77 \\
\hline$n_{0}$ & 14.00 & -3.71 & 14.26 & -2.53 & 2.02 & 5.76 & 1.42 & 9.23 \\
\hline $0.5 n_{0}-n_{0}$ & 14.47 & -0.48 & 14.57 & -0.41 & 2.17 & 13.61 & 1.50 & 15.38 \\
\hline $0.1 n_{0}-n_{\mathrm{t}}$ & 15.09 & 3.78 & 14.97 & 2.32 & 2.46 & 28.80 & 1.65 & 26.92 \\
\hline Max. diff. & 1.09 & & 0.71 & & 0.55 & & 0.35 & \\
\hline \multicolumn{9}{|l|}{ NL3 $\omega \rho \rho$} \\
\hline unified & 13.42 & & 13.75 & & 2.02 & & 1.43 & \\
\hline$n=0.0$ & 13.51 & 0.67 & 13.81 & 0.44 & 2.11 & 4.46 & 1.49 & 4.20 \\
\hline$n_{\mathrm{c}}$ & 13.5 & 1.12 & 13.85 & 0.73 & 2.18 & 7.92 & 1.53 & 6.99 \\
\hline$n_{\mathrm{t}}$ & 13.5 & 0.60 & 13.8 & 0.36 & 2.1 & 3.96 & 1.48 & 3.50 \\
\hline$n_{0}$ & 13.49 & 0.52 & 13.8 & 0.36 & 2.1 & 3.96 & 1.48 & 3.50 \\
\hline $0.5 n_{0}-n_{0}$ & 13.51 & 0.67 & 13.81 & 0.44 & 2.11 & 4.46 & 1.49 & 4.20 \\
\hline $0.1 n_{0}-n_{\mathrm{t}}$ & 13.49 & 0.52 & 13.8 & 0.36 & 2.1 & 3.96 & 1.48 & 3.50 \\
\hline Max. diff. & 0.15 & & 0.10 & & 0.16 & & 0.10 & \\
\hline
\end{tabular}

(7) $n_{1}=0.1 n_{0}, n_{2}=n_{\mathrm{t}}$ : a low matching of the EOS is considered. The DH EOS is used for $n<0.1 n_{0}$ and the core EOS above $n_{\mathrm{t}}$.

If the matching is defined at a given density $n_{\mathrm{m}}=n_{1}$, the gluing is done imposing $P_{2}=P_{1}$. The curves do not coincide because the matching has been performed in different ways. While the maximum mass allowed for a stable star is not affected by the chosen crust-core matching, the same is not true for the radius and crust thickness of stars with a standard mass of $\sim 1.4 M_{\odot}$. The two EOS considered in this example for the crust and the core have quite different properties at saturation density, in particular for the density dependence of the symmetry energy; see Table II. This situation is, however, common in the literature. In fact, the GM1 EOS was parametrized to describe both nuclear saturation properties and neutron star properties.

In Table I, the radius and crust thickness of 1.0 and $1.4 M_{\odot}$ NS are given for three models, GM1, NL3 [39], and NL3 $\omega \rho$ [40], and several matching schemes, together with relative differences with respect to the value for the unified EOS.

As expected the crust-core matching affects more strongly the less massive stars. Depending on the matching procedure, 
the differences in the radius and the crust thickness for a $1.0 M_{\odot}$ star can be as large as $\sim 1$ and $\sim 0.5 \mathrm{~km}$, respectively. This corresponds to relative differences as large as $\sim 4 \%$ for the radius and $\sim 30 \%$ for the crust thickness. This is to be compared with the expected precision of $\sim 5 \%$ on the radius measurement from future x-ray telescopes (NICER, Athena, ... ). Similarly the crust thickness differs by $\sim 0.5 \mathrm{~km}$ depending on the gluing. This quantity is particularly important for the study of the thermal relaxation of accreting NS $[41,42]$, the glitch phenomenon $[17,43,44]$, the torsional crustal vibrations, and the maximum quadrupole ellipticity sustainable by the crust [45].

The differences between matchings are much smaller if the NL3 $\omega \rho$ core EOS is considered, because this model has nuclear matter saturation properties similar to the ones of the SLy4 parametrization [15] used in the DH EOS.

\section{B. Matching and thermodynamic inconsistency}

Two basic methods can be used in order to match two EOS for the crust and the core: the first based on the $P(n)$ relation and the second on the $P(\rho)$ function.

The first method consists of treating the baryon number density as an independent variable. Consider an EOS for the crust, $P_{\mathrm{cr}}(n)$ and $\rho_{\mathrm{cr}}(n)$, and another one for the core, $P_{\mathrm{co}}(n)$ and $\rho_{\mathrm{co}}(n)$.

Let us assume that the matching region lies between two densities, $n_{1}$ and $n_{2}>n_{1}$. First let us build the matched $P(n)$ function. For $n<n_{1}, P(n)=P_{\mathrm{cr}}(n)$ and for $n>n_{2}, P(n)=$ $P_{\text {co }}(n)$. In the matching region, one can assume a form (usually linear or logarithmic) for the function $P(n)$ such that $P\left(n_{1}\right)=$ $P_{1}=P_{\mathrm{cr}}\left(n_{1}\right)$ and $P\left(n_{2}\right)=P_{2}=P_{\mathrm{co}}\left(n_{2}\right)$.

Then one needs to build the function $\rho(n)$. For $n<n_{1}$, $\rho(n)=\rho_{\text {cr }}(n)$. Let us define the chemical potential at the density $n_{1}: \mu_{1}=\left[P_{1}+\rho_{\mathrm{cr}}\left(n_{1}\right)\right] / n_{1}$. By imposing thermodynamic consistency, the value of chemical potential $\mu$ at a density $n$ in the matching region can be derived using the $P(n)$ relation:

$$
\mu(n)=\mu_{1}+\int_{n_{1}}^{n} \frac{d P(n)}{n} .
$$

The matched energy density equals

$$
\rho(n)=n \mu(n)-P(n) .
$$

However this technique generally leads to thermodynamic inconsistency with the core EOS: the value of chemical potential $\mu_{2}$ at the density $n_{2}$ obtained from Eq. (6) differs from $\mu_{\mathrm{co}}\left(n_{2}\right)=\left[P_{2}+\rho_{\mathrm{co}}\left(n_{2}\right)\right] / n_{2}$. As a consequence $\rho\left(n_{2}\right)$ given by Eq. (7) is different from $\rho_{\mathrm{co}}\left(n_{2}\right)$. In order to get a thermodynamically consistent EOS for $n>n_{2}$ one has to add a constant value (an energy shift):

$$
\Delta \mu=\mu\left(n_{2}\right)-\mu_{\mathrm{co}}\left(n_{2}\right)
$$

to the chemical potential in the core. Then the energy density $\rho(n)$ for $n>n_{2}$ is

$$
\rho(n)=\rho_{\mathrm{co}}(n)+n \Delta \mu .
$$

Of course, such a procedure affects the whole EOS for the core, but the main effect on the $M(R)$ relation is for NS with a central pressure close to $P_{2}$.
The second method considers the energy density $\rho$ as an independent variable. This can be motivated by the TOV equations since this quantity and the function $P(\rho)$ actually enter the stress-energy tensor in the Einstein equations. Thus the EOS can be written in the form $P_{\mathrm{cr}}(\rho)$ and $n_{\mathrm{cr}}(\rho)$ for the crust and $P_{\mathrm{co}}(\rho)$ and $n_{\mathrm{co}}(\rho)$ for the core. The matching region is defined such that $\rho_{1}<\rho<\rho_{2}$.

The first step consists of obtaining the function $P(\rho)$. For $\rho<\rho_{1}, P(\rho)=P_{\mathrm{cr}}(\rho)$ and for $\rho>\rho_{2}, P(\rho)=P_{\mathrm{co}}(\rho)$. Similarly to the first method one can assume a form for the function $P(\rho)$ in the matching region such that $P\left(\rho_{1}\right)=P_{1}=$ $P_{\mathrm{cr}}\left(\rho_{1}\right), P\left(\rho_{2}\right)=P_{2}=P_{\mathrm{co}}\left(\rho_{2}\right)$.

Then one wants to derive the relation $n(\rho)$. For $\rho<\rho_{1}$ one has $n(\rho)=n_{\mathrm{cr}}(\rho)$. Let us define $n_{1}=n_{\mathrm{cr}}\left(\rho_{1}\right)$. Assuming thermodynamic consistency, in the matching region, i.e., $\rho_{1} \leqslant$ $\rho<\rho_{2}$, one gets

$$
n(\rho)=n_{1} \exp \left(\int_{\rho_{1}}^{\rho} \frac{d \rho}{P(\rho)+\rho}\right) .
$$

However, as for the first method, this construction does not ensure that $n\left(\rho_{2}\right)$ obtained from the previous formula is equal to $n_{\text {co }}\left(\rho_{2}\right)$. A similar conclusion can be reached for the chemical potential at $\rho_{2}$. Thus one has to modify the $n(\rho)$ dependence for the core EOS in order to ensure thermodynamic consistency. For $\rho>\rho_{2}$, the matched EOS is

$$
n(\rho)=n_{\mathrm{co}}(\rho) \frac{n\left(\rho_{2}\right)}{n_{\mathrm{co}}\left(\rho_{2}\right)} .
$$

This approach does not affect the $P(\rho)$ relation (nor the gravitational mass and the radius), but strictly speaking the microscopic model of dense matter is changed since it is the baryon number density which is the basic quantity for the theoretical calculations, within the many-body theory, of dense matter properties. Of course the accepted procedure given by Eq. (11) also influences the value of a baryon chemical potential (dividing it by the same factor).

\section{Thermodynamic consistency and causality}

In principle, when gluing two EOS one should match all thermodynamic quantities: the pressure $P$, the energy density $\rho$, and the baryonic density $n$. In other words, a pair of functions for the pressure and the energy density should be constructed so that thermodynamic consistency is fulfilled.

Let us consider the EOS for the core and the crust, this time in terms of the chemical potential $\mu, P_{\mathrm{cr}}(\mu)$ in the crust and $P_{\mathrm{co}}(\mu)$ in the core. The matching region is defined by $\mu_{1}<\mu<\mu_{2}$. Let us define $P_{\mathrm{cr}}\left(\mu_{1}\right)=P_{1}$ and $P_{\mathrm{co}}\left(\mu_{2}\right)=P_{2}$. The function $P(\mu)$ in the matching region and its first derivative, which is the baryon number density $n$, should fulfill the conditions of continuity given by

$$
P\left(\mu_{1}\right)=P_{1}, \quad P\left(\mu_{2}\right)=P_{2} .
$$

Thermodynamic consistency and causality imply that the following conditions on the derivatives must be fulfilled in the matching region:

(1) $n$ is an increasing function of $P$, i.e., $P(\mu)$ is increasing and convex; 


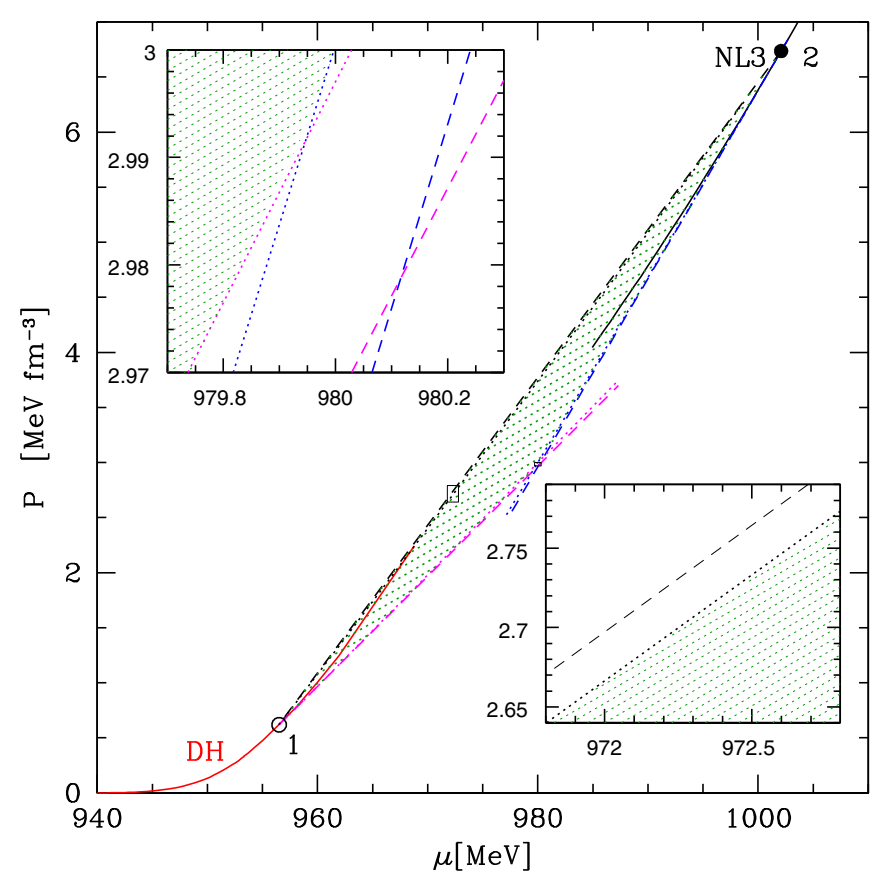

FIG. 2. Pressure $P$ versus chemical potential $\mu$, for the NL3 EOS for the core (black solid line) and DH for the crust EOS (red solid line). The presented situation corresponds to a matching between $n_{1}=$ $0.09 \mathrm{fm}^{-3}$ and $n_{2}=n_{0}=0.16 \mathrm{fm}^{-3}$. The dashed lines correspond to the condition of thermodynamical consistency and are given by Eqs. (14) and (15). The dotted lines are given by the causality limit, Eqs. (16) and (17). The area defined by these lines corresponds to the shaded region and is a bit smaller than the region allowed for a thermodynamically consistent matching between points 1 and 2 (see insets).

(2) $(d P / d \rho)^{1 / 2}=v_{\text {sound }} / c \leqslant 1$, with the energy density $\rho(\mu)=n(\mu) \mu-P(\mu)$.

From the first requirement one can derive a necessary condition (using Lagrange's mean value theorem),

$$
\begin{aligned}
n_{1} & <\frac{P_{2}-P_{1}}{\mu_{2}-\mu_{1}}<n_{2} \\
\text { with } n_{1} & =\left(\frac{d P_{\mathrm{cr}}}{d \mu}\right)_{\mu_{1}} \text { and } n_{2}=\left(\frac{d P_{\mathrm{co}}}{d \mu}\right)_{\mu_{2}} .
\end{aligned}
$$

If the above inequality is not fulfilled, a matching of the crust and core EOS using a continuous $P(\mu)$ function cannot be obtained.

Figure 2 shows an example of the matching between the DH EOS for the crust and the NL3 EOS for the core, with $n_{1}=0.09 \mathrm{fm}^{-3}$ and $n_{2}=n_{0}$. The points 1 and 2 have the coordinates $\left(n_{1}, P_{1}\left(\mu_{1}\right)\right)$ and $\left(n_{2}, P_{2}\left(\mu_{2}\right)\right)$, respectively.

Any thermodynamically consistent EOS is located in the triangle defined by the two tangents at the points 1 and 2 ,

$$
P=P_{1}+n_{1}\left(\mu-\mu_{1}\right), \quad P=P_{2}+n_{2}\left(\mu-\mu_{2}\right)
$$

and the straight line connecting these two points,

$$
P=P_{1}+\left(P_{2}-P_{1}\right) \frac{\mu-\mu_{1}}{\mu_{2}-\mu_{1}} .
$$

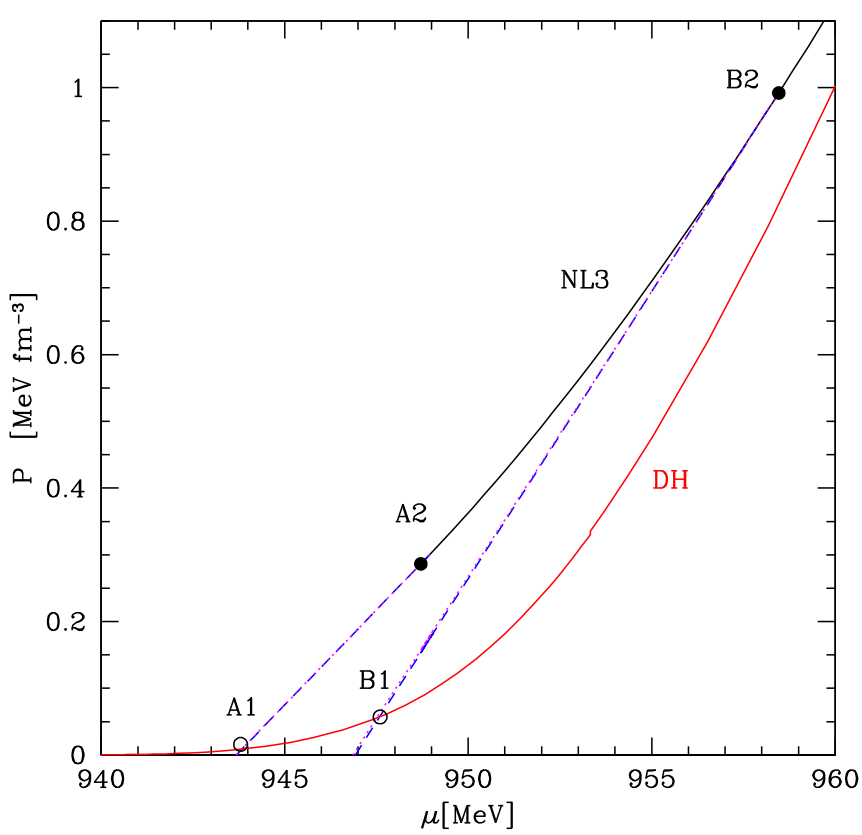

FIG. 3. Pressure $P$ versus chemical potential $\mu$, for the NL3 EOS for the core (black solid line) and DH for the crust EOS (red solid line). The points $\mathrm{A} 2$ and $\mathrm{B} 2$ correspond to two different values of $n_{2}: n_{\mathrm{t}}$, the core-crust transition density, and $n_{0} / 2$, respectively. The dashed lines indicate the condition of thermodynamical consistency and the dotted lines mark the causality limit. They are almost indistinguishable. The points $\mathrm{A} 1$ and $\mathrm{B} 1$ correspond to the higher limits on $\mu$ or equivalently $n$, such that a thermodynamically consistent gluing with the core at the points $\mathrm{A} 2$ and $\mathrm{B} 2$ exists.

However the bounds defined by Eqs. (14-15) describe incompressible matter with a constant baryon number density equal to $n_{1}, n_{2},\left(P_{2}-P_{1}\right) /\left(\mu_{2}-\mu_{1}\right)$, respectively.

The additional constraint resulting from the causality requirement reduces the allowed region, but the change is very small (see zoomed inserts in Fig. 2). Instead of the tangents at the points $\mathbf{1}$ and $\mathbf{2}$ given by Eq. (14) the causality limit corresponds to

$$
P=P_{i}+n_{i}\left(\mu-\mu_{i}\right) \frac{\mu+\mu_{i}}{2 \mu_{i}}, \quad i=1,2 .
$$

The line connecting the two points and fulfilling the causality condition is

$$
P=P_{1}+\left(P_{2}-P_{1}\right) \frac{\mu^{2}-\mu_{1}^{2}}{\mu_{2}^{2}-\mu_{1}^{2}} .
$$

In Fig. 3 the matching conditions for the same EOS as in Fig. 2 but for lower $n_{2}$ (or equivalently $\mu_{2}$ ) are presented. The points $\mathrm{A} 2$ and $\mathrm{B} 2$ correspond to $n_{2}=n_{\mathrm{t}}$, the transition density between the core and the crust, and $n_{2}=n_{0} / 2$, respectively. In these cases, for a given $n_{2}$ two upper limits on $n_{1}$ can be obtained: the first one by the crossing point between the tangent at the point 2 given by Eq. (13) and the crust EOS, and the second one by the intersection of the line defined by Eq. (16) and the crust EOS. Here both upper limits are actually almost 
identical and correspond to the points $\mathrm{A} 1$ and $\mathrm{B} 1$, obtained for the points $\mathrm{A} 2$ and $\mathrm{B} 2$, respectively.

For the matching of the NL3 EOS with the DH crust if we choose $n_{2}=n_{0} / 2$ (point $B_{2}$ in the figure) then the condition given by Eqs. (13)-(16) results in $n_{1}<0.02 \mathrm{fm}^{-3}$ (point $B_{1}$ ). It means that the matching region in terms of $n$ and $P$ should be extremely large, with $n_{2}>4 n_{1}$ and $P_{2}>16 P_{1}$.

A similar estimation for the point $A_{2}$ with $n_{2}=n_{\mathrm{t}}$ results in $n_{1}<0.0075 \mathrm{fm}^{-3}$ (upper limit marked by the point $A_{1}$ ) and the matching described in Sec. III A for the NL3 model $\left(n_{1}=\right.$ $0.1 n_{0}, n_{2}=n_{\mathrm{t}}$ ) cannot be performed in a thermodynamically consistent and causal way, unless one changes the core EOS, as described in Sec. III B. As a consequence not all matchings presented in Sec. IIIB are thermodynamically consistent and/or causal.

It should be mentioned that limits defined by Eqs. (14)-(17) correspond to a very high stiffness of matter [incompressible fluid for Eqs. (14) and (15) and sound speed equal to the speed of light for Eqs. (16) and (17)]. In reality the sound speed in the matching region is much smaller. Assuming linear dependence between the pressure and energy density in this region, $P=a(\rho-\tilde{\rho})$, with $a$ being the square of sound speed (in the units of $c$ ) we obtain a general formula for the $P(\mu)$ dependence which is thermodynamically consistent:

$$
P=P_{1}+\left(P_{2}-P_{1}\right) \frac{\mu^{b}-\left(\mu_{1}\right)^{b}}{\left(\mu_{2}\right)^{b}-\left(\mu_{1}\right)^{b}}, \quad b=\frac{1+a}{a} .
$$

Then Eqs. (15) and (17) are special cases of Eq. (18) for $a=\infty$ and $a=1$, respectively. Thermodynamically correct linear matching for a given $a$ generally leads to density discontinuities (first-order phase transitions) at $P=P_{1}$ and $P=P_{2}$ (for details see the Appendix). There exists a minimum value of $a\left(a_{\min }\right)$ for which such a procedure is possible (with only one density jump) and in general it is larger than the slope of the line connecting points $\left(\rho_{1}, P_{1}\right)$ and $\left(\rho_{2}, P_{2}\right)$ (equality being reached for a very specific relation between $P, \rho$, and $\mu$ at the boundaries of a matching region).

In Fig. 4 we visualize the crucial role of the thermodynamic consistency for a determination of the stellar radius. For a thermodynamically consistent matching between core and crust, the radii of NS models with $M>1.0 M_{\odot}$ are very similar even for a very different matching functions (e.g., for $a=0.33$ and $a=0.0913)$. For a simplest $P(\rho)$ matching (linear function without density jumps) we get $a=\left(P_{2}-P_{1}\right) /\left(\rho_{2}-\rho_{1}\right)=$ 0.088; smaller than the minimum value $a_{\min }=0.0913$ allowed by the condition of thermodynamical consistency (for details see Appendix). The requirement that the matching function $P(\rho)$ is continuous at $P_{1}$ and $P_{2}$ results in a discontinuity in the $\mu(P)$ function (i.e., a shift in the baryon chemical potential $\mu)$ and leads to thermodynamical inconsistency.

\section{UNIFIED EQUATIONS OF STATE}

We introduce a set of unified EOS which were built within a RMF approach or using nonrelativistic Skyrme interactions. The choice of models takes into account the astrophysical constraints on the maximum NS mass and the speed of sound:

$$
M_{\max } \geqslant 2 M_{\odot}, \quad v_{\text {sound }}\left(2 M_{\odot}\right)<c .
$$
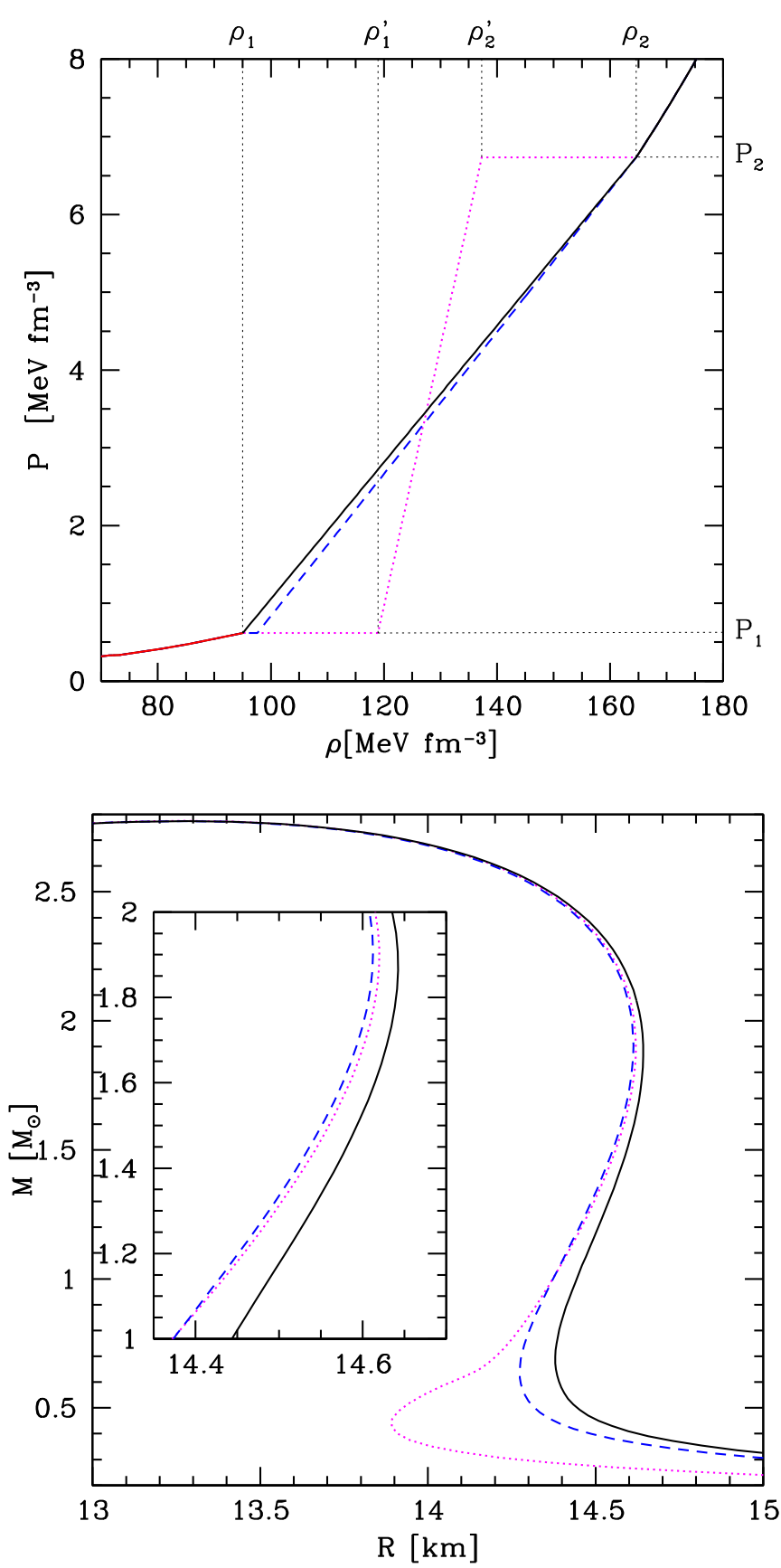

FIG. 4. Linear matching between core and crust. Upper panel, solid line: direct connection between $\left(\rho_{1}, P_{1}\right)$ and $\left(\rho_{2}, P_{2}\right)$ which is thermodynamically inconsistent $(a=0.088)$; dotted and dashed lines: thermodynamically consistent gluing for $a=0.33$ (dotted) and $a=a_{\min }=0.0913$ (dashed) accompanied by a density jump (jumps) defined by $\rho_{1}^{\prime}, \rho_{2}^{\prime}$ (dotted line). Bottom panel: $M(R)$ for these matchings; thermodynamically consistent solutions give very similar radii for $M>1.0 M_{\odot}$. Stars with central pressure equal to $P_{2}$ have masses $\sim 0.6 M_{\odot}$.

To these two constraints we will add experimental and theoretical constraints on nuclear matter properties and we will discuss the uncertainty on the determination of the radius and the crust thickness of $1.0 M_{\odot}, 1.4 M_{\odot}$, and $1.8 M_{\odot}$ stars. 
TABLE II. Nuclear and astrophysical properties of the RMF models. Energy per nucleon $\left(E_{\mathrm{s}}\right)$, compression modulus $(K)$, symmetry energy $(J)$, slope $(L)$, and incompressibility $\left(K_{\text {sym }}\right)$ of the symmetry energy at the saturation point of uniform symmetric nuclear matter of density $n_{\mathrm{s}}$. The density at the edge of the liquid uniform core is denoted as $n_{\mathrm{t}}$. In the column "Pasta," the type(s) of pasta phase in the bottom layer (mantle) above the edge of the core is indicated: $s$ stands for slab, $r$ for rod, and d for droplet phases. $M_{\max }^{\text {noY }}$ is the maximum mass for a purely nucleonic core composition. $n_{\mathrm{DU}}$ and $M_{\mathrm{DU}}$ are respectively the baryon density and NS mass threshold above which the nucleonic DUrca is switched on for a purely nucleonic core.

\begin{tabular}{|c|c|c|c|c|c|c|c|c|c|c|c|c|}
\hline Model & $\begin{array}{c}n_{\mathrm{s}} \\
\left(\mathrm{fm}^{-3}\right)\end{array}$ & $\begin{array}{c}E_{\mathrm{s}} \\
(\mathrm{MeV})\end{array}$ & $\begin{array}{c}K \\
(\mathrm{MeV})\end{array}$ & $\begin{array}{c}J \\
(\mathrm{MeV})\end{array}$ & $\begin{array}{c}L \\
(\mathrm{MeV})\end{array}$ & $\begin{array}{c}K_{\text {sym }} \\
(\mathrm{MeV})\end{array}$ & $\begin{array}{c}n_{\mathrm{t}} \\
\left(\mathrm{fm}^{-3}\right)\end{array}$ & Pasta & $\begin{array}{l}M_{\max }^{\mathrm{noY}} \\
\left(M_{\odot}\right)\end{array}$ & $\begin{array}{c}n_{\mathrm{DU}} \\
\left(\mathrm{fm}^{-3}\right)\end{array}$ & $\begin{array}{l}M_{\mathrm{DU}} \\
\left(M_{\odot}\right)\end{array}$ & Ref. \\
\hline NL3 & 0.149 & -16.2 & 271.6 & 37.4 & 118.9 & 101.6 & 0.056 & $\mathrm{~d}$ & 2.77 & 0.20 & 0.84 & [39] \\
\hline TM1 & 0.146 & -16.3 & 281.2 & 36.9 & 111.2 & 33.8 & 0.058 & $\mathrm{~d}$ & 2.18 & 0.21 & 0.81 & [46] \\
\hline $\mathrm{DDH} \delta$ & 0.153 & -16.3 & 240.3 & 25.6 & 48.6 & 91.4 & 0.080 & $\mathrm{~s}, \mathrm{r}, \mathrm{d}$ & 2.14 & 0.58 & 1.90 & [47] \\
\hline BSR2 & 0.149 & -16.0 & 239.9 & 31.5 & 62.0 & -3.1 & 0.065 & $\mathrm{~s}, \mathrm{r}, \mathrm{d}$ & 2.38 & 0.37 & 1.61 & {$[48,49]$} \\
\hline NL3 $\omega \rho$ & 0.148 & -16.2 & 271.6 & 31.7 & 55.5 & -7.6 & 0.082 & $\mathrm{~s}, \mathrm{r}, \mathrm{d}$ & 2.75 & 0.50 & 2.55 & [40] \\
\hline DDME2 & 0.152 & -16.1 & 250.9 & 32.3 & 51.2 & -87.1 & 0.072 & $\mathrm{~s}, \mathrm{r}, \mathrm{d}$ & 2.48 & & & [50] \\
\hline DD2 & 0.149 & -16.0 & 242.6 & 31.7 & 55.0 & -93.2 & 0.067 & $\mathrm{~s}, \mathrm{r}, \mathrm{d}$ & 2.42 & & & [51] \\
\hline
\end{tabular}

Some of the proposed EOS, namely the RMF EOS, are not fully unified since the outer crust is not calculated within the framework of the model that defines the rest of the EOS, but we have checked that since most of the outer crust is defined by experimental results, the use of other EOS for the outer crust, such as $[23,24]$, does not significantly affect the star radius with a mass above $1.0 M_{\odot}$.

\section{A. RMF unified EOS}

In the present study we consider two different types of models within the relativistic mean field (RMF) approach: (i) nonlinear Walecka models (NLWM) with constant coupling parameters, and (ii) density-dependent hadronic models (DDH) with density-dependent coupling parameters. The only condition that has been imposed a priori is that the models describe a $2 M_{\odot}$ star. Within the first category a set of models that span a quite large range of nuclear saturation properties was chosen: NL3 [39] with a large symmetry energy slope and incompressibility at saturation and which was fitted to the ground state properties of both stable and unstable nuclei; NL3 $\omega \rho$ [40] which, compared to NL3, has a softer density dependence of the symmetry energy through the inclusion of a nonlinear $\omega \rho$ term; GM1 [37] fitted to describe nuclear matter saturation properties subject to NS mass-radius constraints; TM1 [46] which includes nonlinear $\omega$ meson terms in order to soften the matter at high densities and is one of the classical supernova EOS [52,53]; and two parametrizations, BSR2 and BSR6, with several nonlinear terms mixing the $\omega, \rho$, and $\sigma$ mesons [48,49]. Within the second type, three EOS were considered: DDME2 [50], DD2 [54], and DDH $\delta$ [47], the last one also including the $\delta$ meson. Some properties of the set of models we use are indicated in Table II.

We have built unified EOS for these models in the following way: (a) for the outer crust we take the EOS proposed in [24]; (b) for the inner crust we perform a Thomas-Fermi calculation and allow for nonspherical clusters according to [38,55]; (c) for the core we consider the homogeneous matter EOS. The transition between the inner crust and the core is smooth. The maximum mass stars in Table II have been obtained with the unified EOS.

Two compositions are considered: purely nucleonic and baryonic matter with both nucleons and hyperons. The nucleonic models, the so-called noY models, include the scalar $\sigma$, vector $\omega$, and vector-isovector $\rho$ meson fields (possibly also the $\delta$ meson) together with the nucleon doublet: neutron $n$ and proton $p$. The $\mathbf{Y}$ and Yss models denote hyperonic EOS and, with respect to the noY models, they also include the six lightest hyperons $\left(\Lambda^{0}\right.$, the $\Sigma^{+}, \Sigma^{0}, \Sigma^{-}$triplet, and the $\Xi^{0}, \Xi^{-}$doublet) and the hidden-strangeness vector-isoscalar $\phi$ meson for the $\mathbf{Y}$ models, or the $\phi$ meson together with the hidden-strangeness scalar-isoscalar $\sigma^{*}$ for the Yss models.

The vector meson-hyperon coupling constants are always calculated assuming SU(6) symmetry (see, e.g., [56-58]):

$$
\begin{aligned}
\frac{1}{3} g_{\omega N} & =\frac{1}{2} g_{\omega \Lambda}=\frac{1}{2} g_{\omega \Sigma}=g_{\omega \Xi}, \\
2 g_{\phi \Lambda} & =2 g_{\phi \Sigma}=g_{\phi \Xi}=-\frac{2 \sqrt{2}}{3} g_{\omega N}, \\
g_{\rho N} & =\frac{1}{2} g_{\rho \Sigma}=g_{\rho \Xi}, \\
g_{\phi N} & =0, \\
g_{\rho \Lambda} & =0,
\end{aligned}
$$

where $N$ stands for nucleons. For models with the density dependent couplings, we define the hyperon-meson couplings as $g_{i Y}(n)=x_{i Y} g_{N Y}(n)$ (see [59]), where $n$ is the total baryonic density and the ratios $x_{i Y}$ are constants. The $g_{\sigma Y}$ couplings, where $Y$ stands for hyperons $(Y=\Lambda, \Sigma, \Xi)$, are obtained from the hyperon potential in symmetric nuclear matter, $U_{Y}^{(N)}$,

$$
U_{Y}^{(N)}=-\left(g_{\sigma Y}+g_{\sigma Y}^{\prime} \rho_{s}\right) s_{0}+\left(g_{\omega Y}+g_{\omega Y}^{\prime} n_{\mathrm{s}}\right) w_{0},
$$

with $s_{0}$ and $w_{0}$ the mean-field values of the $\sigma$ and $\omega$ meson fields, respectively, and $\rho_{s}$ the scalar density, all quantities calculated at saturation density. The terms involving the derivatives of the couplings with respect to the total density 
only occur for models with density dependent couplings. Here we adopt the following values at saturation density: $U_{\Lambda}^{(N)}\left(n_{\mathrm{s}}\right)=$ $-28 \mathrm{MeV}, U_{\mathrm{\Xi}}^{(N)}\left(n_{\mathrm{s}}\right)=-18 \mathrm{MeV}$, and $U_{\Sigma}^{(N)}\left(n_{\mathrm{s}}\right)=30 \mathrm{MeV}$ [60] (see also the discussion in [61]).

In the Yss model the $\sigma^{*}$ meson is also included. It is assumed that it does not couple to a nucleon, i.e., $g_{\sigma^{*} N}=0$. The $\Lambda$ potential in $\Lambda$ matter is given by

$$
U_{\Lambda}^{(\Lambda)}=-g_{\sigma \Lambda} s_{0}-g_{\sigma^{*} \Lambda} s_{0}^{*}+g_{\omega \Lambda} w_{0}+g_{\phi \Lambda} f_{0},
$$

with $s_{0}^{*}$ and $f_{0}$ the mean-field values of the $\sigma^{*}$ and $\phi$ meson fields, respectively. Taking $U_{\Lambda}^{(\Lambda)}\left(n_{\mathrm{s}}\right)=-5 \mathrm{MeV}$ [62] (see also the discussion in [61]), the value of $g_{\sigma^{*} \Lambda}$ can then be fixed. This is, however, only an indicative effect that should be taken with care, since the hyperon-meson couplings should be constrained by fitting the experimental binding energy of hyperons in hypernuclei [63]. This subject requires further investigation. Since the definition of the hyperon-meson couplings in density dependent couplings involves several choices, we considered that presenting results including the $\sigma^{*}$ meson would introduce further uncertainties and, therefore, decided not to show results with the $\sigma^{*}$ meson for these models. The two remaining coupling constants can be derived taking $g_{\sigma^{*} \Sigma}=g_{\sigma^{*} \Lambda}$ and assuming that $U_{\Xi}^{(\Xi)} \simeq 2 U_{\Lambda}^{(\Lambda)}$. The $\Xi$ potential, $U_{\Xi}^{(\Xi)}$, in symmetric $\Xi^{0}-\Xi^{-}$matter is given by an expression similar to Eq. (21), replacing $\Lambda$ by $\Xi$. For DDH $\delta$ we only present the $\mathbf{Y}$ results because even in this case the maximum mass is far from $2 M_{\odot}$, and the presence of the $\delta$ meson brings extra unknowns in the definition of the hyperon-meson couplings.

Unified EOS are built for all the models and the TOV equations solved. The mass-radius relations $M-R$ of all models, nucleonic and hyperonic, are plotted in Fig. 5. The models were chosen such that nucleonic EOS predict stars with a mass above $2 M_{\odot}$. We can observe that the same occurs for all Y models, except for the one built with $\mathrm{DDH} \delta$. With respect to the Yss models, only the ones built with NL3 or NL3 $\omega \rho$ satisfy the constraint set by PSR J0348+0432. Although models have been distributed between two figures so that they are not too crowded, it is still possible to see that the radius of a $1.5 M_{\odot}$ star varies between $\sim 12.6$ and $14.6 \mathrm{~km}$. Another conclusion is that the onset of hyperons occurs for a mass $\sim 1.4 M_{\odot}$ or above, except for the DDH $\delta$ model.

Models with the largest values of $L$ only predict droplet-like clusters in the inner crust, in accordance with the results of [64]; see Table II.

In Table III we gather some of the properties of the hyperonic stars, including the central baryonic density, the maximum mass, the onset density of each hyperon and the corresponding mass of the star. It is seen that, for the choice of meson-hyperon couplings described above, the first hyperon to set in in all models is the $\Lambda$ meson. The second hyperon is the $\Xi^{-}$hyperon, again in all models. This hyperon is favored with respect to $\Sigma^{-}$because of the attractive $\Xi$ potential in nuclear matter. The third meson to set in, when it exists in a stable NS, is either $\Sigma^{-}$or $\Xi^{0} . \Sigma^{-}$appears only when the $\sigma^{*}$ meson is not included in the calculation, because the attractive effect of the $\sigma^{*}$ meson is stronger for $\Xi$ hyperons due to their double strangeness charge.
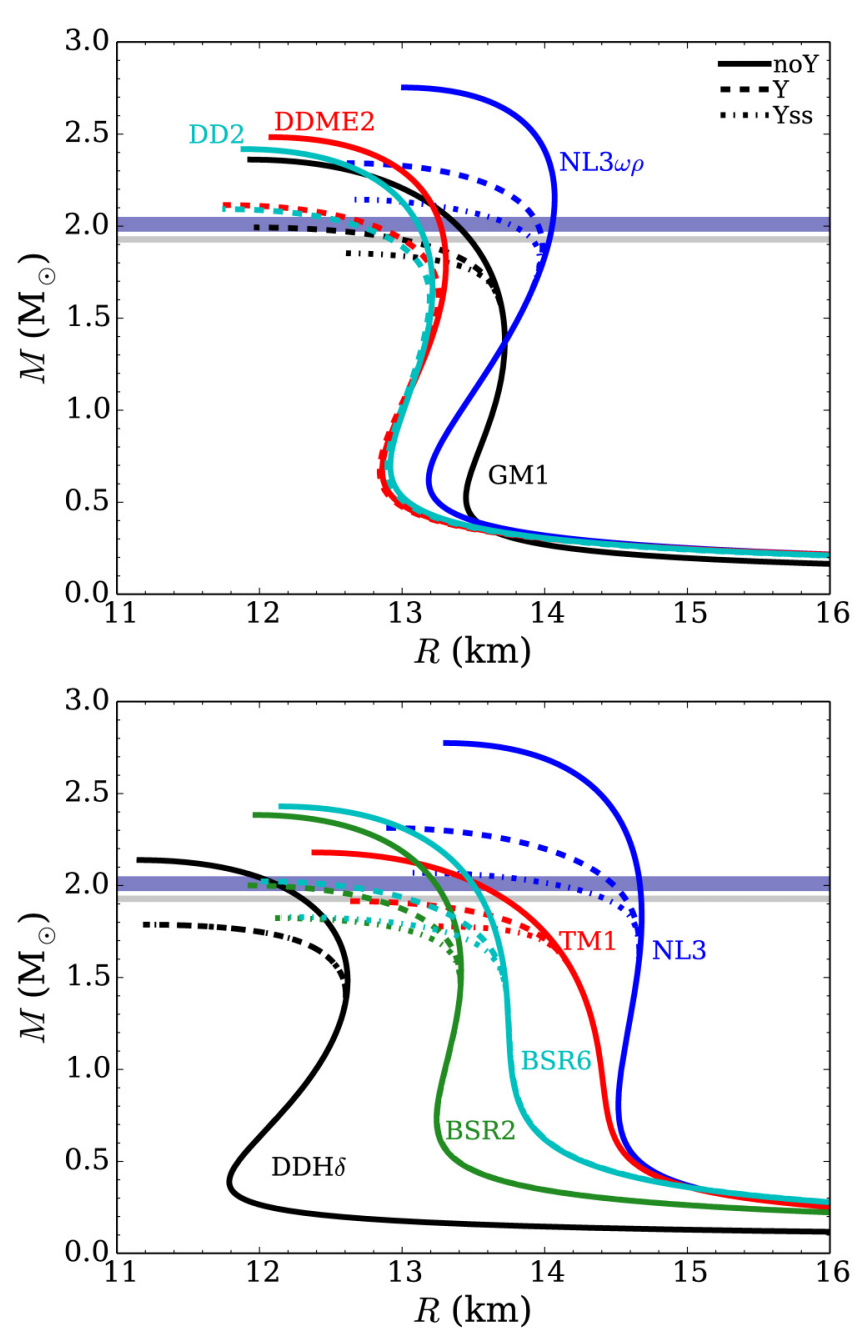

FIG. 5. Mass-radius relation for the various RMF models: noY, $\mathbf{Y}$, and Yss. The horizontal lines indicate the constraints set by the pulsars PSR J0348+0432 and PSR J1614- 2230.

\section{B. Nonrelativistic unified EOS}

To construct nonrelativistic unified equations of state we proceed as follows. We select a large set of different Skyrme functionals proposed in the recent nuclear physics literature. At low density we variationally determine the nucleus $A$ and $Z$ number, as well as the volume $V_{W S}$ of the Wigner-Seitz cell and the density of the free neutron component $n_{\mathrm{g}}$ after neutron drip, employing the same Skyrme functional for both the nucleus and the free neutrons [65]. The baryonic part of the Wigner-Seitz cell energy is written as

$$
E_{\mathrm{WS}}\left(A, Z, n_{\mathrm{g}}, V_{\mathrm{WS}}\right)=V_{\mathrm{WS}} \mathcal{E}_{\mathrm{Sky}}+E^{\mathrm{vac}}+\delta E .
$$

Here, $\mathcal{E}_{\text {Sky }}\left(n_{\mathrm{g}}\right)$ is the energy density of homogeneous neutron matter as given from the chosen Skyrme functional, $E^{\mathrm{vac}}(A, Z)$ is the vacuum energy of a nucleus of mass number $A$ and charge $Z$, and the extra term $\delta E=\delta E_{\text {bulk }}+\delta E_{\text {surf }}+\delta E_{\text {Coul }}$ corresponds to the bulk, surface, and Coulomb in-medium modifications.

For the vacuum energy, we employ a compressible liquiddrop (CLDM) parametrization [66]. The coefficients of this 
TABLE III. Properties of hyperonic RMF models. For given EOS and hyperonic model, the central density $n_{\max }\left(\right.$ in $\mathrm{fm}^{-3}$ ) at the maximum mass $M_{\max }\left(\right.$ in $M_{\odot}$ ) is given. The next columns list the type of hyperons $Y_{i}$, the values of the onset densities $n_{Y_{i}}$ (in fm ${ }^{-3}$ ), and the corresponding NS masses: $M_{Y_{i}}$ (in $M_{\odot}$ ). The last two columns indicate the density and mass threshold, $n_{\mathrm{DU}}$ and $M_{\mathrm{DU}}$ respectively above which the nucleonic DUrca process operates.

\begin{tabular}{|c|c|c|c|c|c|c|c|c|c|c|c|c|c|c|}
\hline EOS & Model & $\begin{array}{c}n_{\max } \\
\left(\mathrm{fm}^{-3}\right)\end{array}$ & $\begin{array}{l}M_{\max }^{Y} \\
\left(M_{\odot}\right)\end{array}$ & $Y_{1}$ & $\begin{array}{c}n_{Y_{1}} \\
\left(\mathrm{fm}^{-3}\right)\end{array}$ & $\begin{array}{c}M_{Y_{1}} \\
\left(M_{\odot}\right)\end{array}$ & $Y_{2}$ & $\begin{array}{c}n_{Y_{2}} \\
\left(\mathrm{fm}^{-3}\right)\end{array}$ & $\begin{array}{c}M_{Y_{2}} \\
\left(M_{\odot}\right)\end{array}$ & $Y_{3}$ & $\begin{array}{c}n_{Y_{3}} \\
\left(\mathrm{fm}^{-3}\right)\end{array}$ & $\begin{array}{c}M_{Y_{3}} \\
\left(M_{\odot}\right)\end{array}$ & $\begin{array}{c}n_{\mathrm{DU}} \\
\left(\mathrm{fm}^{-3}\right)\end{array}$ & $\begin{array}{l}M_{\mathrm{DU}} \\
\left(M_{\odot}\right)\end{array}$ \\
\hline NL3 & Yss & 0.77 & 2.07 & $\Lambda^{0}$ & 0.28 & 1.52 & $\Xi^{-}$ & 0.33 & 1.75 & $\Xi^{0}$ & 0.57 & 2.03 & 0.20 & 0.84 \\
\hline \multirow[t]{2}{*}{ NL3 $\omega \rho$} & Yss & 0.80 & 2.14 & $\Lambda^{0}$ & 0.31 & 1.59 & $\Xi^{-}$ & 0.34 & 1.74 & $\Xi^{0}$ & 0.65 & 2.13 & 0.42 & 1.91 \\
\hline & $\mathbf{Y}$ & 0.79 & 2.34 & $\Lambda^{0}$ & 0.31 & 1.58 & $\Xi^{-}$ & 0.34 & 1.78 & $\Sigma^{-}$ & 0.49 & 2.17 & 0.43 & 2.07 \\
\hline DDME2 & $\mathbf{Y}$ & 0.93 & 2.12 & $\Lambda^{0}$ & 0.34 & 1.41 & $\Xi^{-}$ & 0.37 & 1.55 & $\Sigma^{-}$ & 0.41 & 1.66 & & \\
\hline GM1 & $\mathbf{Y}$ & 0.92 & 1.99 & $\Lambda^{0}$ & 0.35 & 1.48 & $\Xi^{-}$ & 0.41 & 1.67 & & & & 0.28 & 1.10 \\
\hline \multirow[t]{2}{*}{ TM1 } & Yss & 0.73 & 1.78 & $\Lambda^{0}$ & 0.35 & 1.52 & $\Xi^{-}$ & 0.39 & 1.63 & $\Xi^{0}$ & 0.72 & 1.78 & 0.21 & 0.81 \\
\hline & $\mathbf{Y}$ & 0.85 & 1.92 & $\Lambda^{0}$ & 0.32 & 1.40 & $\Xi^{-}$ & 0.42 & 1.70 & & & & 0.21 & 0.81 \\
\hline $\mathrm{DDH} \delta$ & $\mathbf{Y}$ & 1.05 & 1.79 & $\Lambda^{0}$ & 0.37 & 1.30 & $\Xi^{-}$ & 0.52 & 1.60 & $\Sigma^{-}$ & 0.70 & 1.74 & 0.62 & 1.69 \\
\hline DD2 & $\mathbf{Y}$ & 0.93 & 2.10 & $\Lambda^{0}$ & 0.37 & 1.52 & $\Xi^{-}$ & 0.38 & 1.55 & $\Sigma^{-}$ & 0.45 & 1.75 & & \\
\hline BSR2 & Yss & 0.84 & 1.84 & $\Lambda^{0}$ & 0.34 & 1.37 & $\Xi^{-}$ & 0.39 & 1.54 & & & & 0.39 & 1.55 \\
\hline
\end{tabular}

mass formula are fitted out of Hartree-Fock calculations in slab geometry, using the same Skyrme effective interaction which is employed for the free neutron component. The absence of shell effects and curvature terms in this analytic parametrization implies that a mass shift which, depending on the Skyrme interaction, can be as high as $0.5 \mathrm{MeV} /$ nucleon, is observed with respect to experimentally measured masses. As a consequence, the EOS of the external part of the outer crust differs from the one we would get employing experimental data. This is true even for recent sophisticated Skyrme functionals which have shown, if full HFB calculations are performed, a very good agreement with experimentally measured nuclear masses [67]. An example is given in Fig. 6, which shows for a representative Skyrme functional the discontinuity in the baryonic pressure obtained if an EOS using experimental masses is matched with the unified prescription.

However, the deviation between the CLDM-based EOS and the one obtained when the experimental mass data are used is small enough to impact the $M(R)$ relation to less than $1 \%$, as one can see in the lower panel of Fig. 6.

The bulk in-medium correction to the nuclear energy $\delta E_{\text {bulk }}$ is approximated by

$$
\delta E_{\text {bulk }}\left(A, Z, n_{\mathrm{g}}\right)=-\frac{A}{n_{\mathrm{s}}} \mathcal{E}_{\mathrm{Sky}},
$$

where $A / n_{\mathrm{s}}(\delta)$ represents the equivalent cluster volume corresponding to the given isospin asymmetry $\delta$, evaluated in the nuclear bulk. For a nucleus in the vacuum we take for the bulk asymmetry the estimation from the droplet model [68],

$$
\delta=\delta_{0}=\left[\left(1-2 \frac{Z}{A}\right)+\frac{3 a_{c}}{8 Q} \frac{Z^{2}}{A^{5 / 3}}\right] /\left(1+\frac{9 J}{4 Q A^{1 / 3}}\right) .
$$

In this equation, $J$ is the symmetry energy per nucleon at the saturation density of symmetric matter, $Q$ is the surface stiffness coefficient, and $a_{\mathrm{c}}$ is the Coulomb parameter. In the presence of an external neutron gas of density $n_{\mathrm{g}}$, the bulk asymmetry defined by Eq. (24) is generalized to account for the contribution of the gas as [69]

$$
\delta\left(A, Z, n_{\mathrm{g}}\right)=\left(1-\frac{n_{\mathrm{g}}}{n_{\mathrm{s}}(\delta)}\right) \delta_{0}+\frac{n_{\mathrm{g}}}{n_{\mathrm{s}}(\delta)},
$$

where $\delta_{0}$ is the asymmetry value given by Eq. (24) considering only the bound part of the cluster. For details, see $[65,69,70]$.

The Coulomb energy shift $\delta E_{\text {Coul }}$ is due to the screening effect of the electrons, and it is evaluated in the standard Wigner-Seitz approximation [36]. The residual energy shift corresponds to the in-medium modification of the surface tension in the inner crust. This term can be evaluated in the extended Thomas-Fermi approximation [70-72]. For the applications of the present paper, this correction has been neglected. The error induced by this approximation on the $M(R)$ relation is quantified below in this section, and shown to be reasonably small. However, this effect, together with the curvature terms which are also neglected, is important for a precise determination of the transition density. For this reason we leave the study of the functional dependence of the transition density to future work.

Since the droplet phase is known to be the dominant pasta phase in $\beta$ equilibrium [33], we have not considered possible deviation from spherical symmetry in the nucleus functional.

The Wigner-Seitz energy density from Eq. (22) is minimized with respect to its arguments with the additional requirement of $\beta$ equilibrium, thus leading to the equilibrium composition of the neutron star crust at each baryonic density value [65]. In the absence of deformation degrees of freedom the crust-core transition occurs via a narrow phase coexistence 

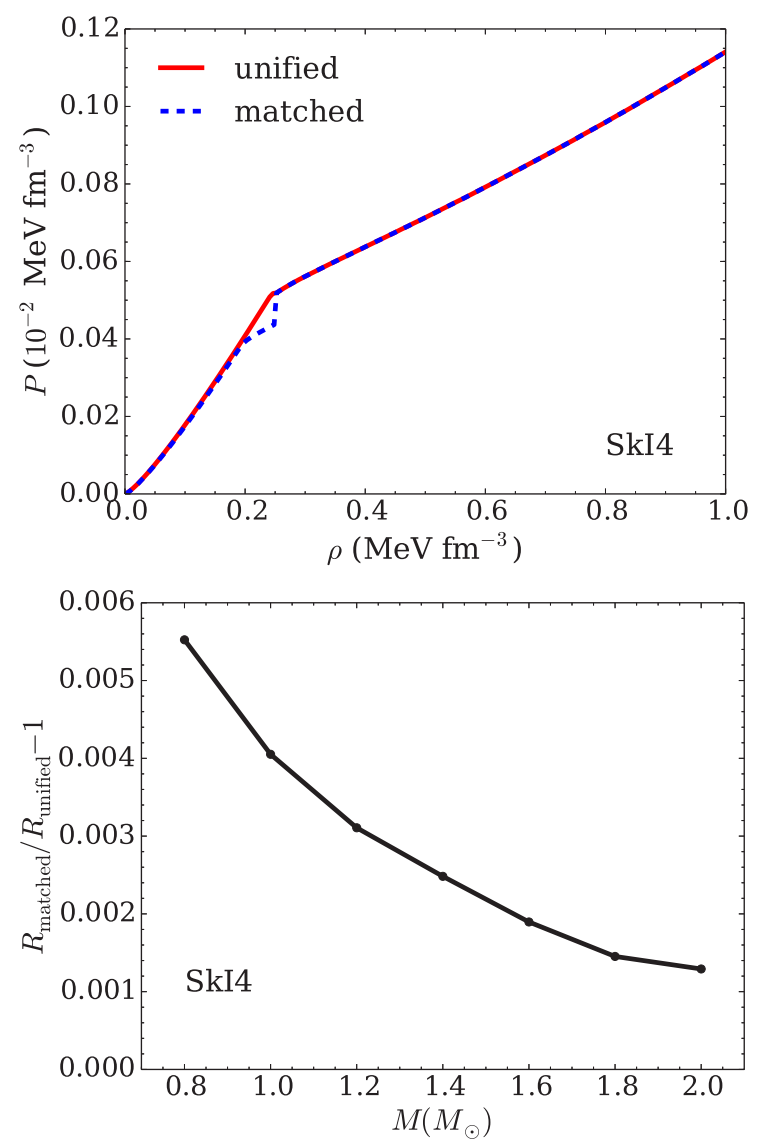

FIG. 6. $(n, p, e)$ matter in $\beta$ equilibrium. Upper panel: total pressure versus total energy density for the unified EOS (solid lines) and using experimental masses when available (dashed lines). Lower panel: relative deviation of the NS radius as a function of mass with the two prescriptions shown on the top part. The SkI4 functional is used.

domain [65]. The transition to the core is defined by the high density border of this first-order phase transition region.

As stated above, a precise treatment of this transition requires proton shell effects, curvature terms, modifications of the surface tension, and deformation degrees of freedom. However the energy density landscape turns out to be extremely flat close to the transition point $[65,71]$. This means that the approximations employed in Eq. (22) prevent a precise determination of the transition density, but do not affect the density behavior of the pressure.

The $M(R)$ relation is then obtained integrating the TOV equations. Only the functionals which produce, without hyperonic degrees of freedom, maximum NS masses of at least, within $1 \%$ accuracy, $2 M_{\odot}$, and which are causal up to the highest densities met in such massive stars are kept for the following analysis. The list of the functionals which have been retained, and the corresponding EOS parameters, are listed in Table IV. With the exception of BSk20 and BSk26 models, the causality condition actually holds also up to the maximum mass. The resulting $M(R)$ relation is shown in Fig. 7. It is seen that $1.0 M_{\odot}-1.5 M_{\odot}$ stars span a radius range $\sim 3 \mathrm{~km}$ wide, from $\sim 11.5$ to $\sim 14.2 \mathrm{~km}$.
In the case of some of the Skyrme functionals developed by the Brussels group [84,85], the $M(R)$ relation has already been calculated with a unified EOS obtained by numerically solving in the crust the full Hartree-Fock-Bogoliubov problem in the Wigner-Seitz cell $[86,87]$. A comparison with our results using the simplified CLDM allows quantifying the error which occurs because of the different approximations employed to get an analytic model, namely the lack of shell effects, curvature terms, and in-medium modifications of the surface tension. This comparison is shown in Fig. 8. We can see that the estimation of the maximum mass is never affected by the approximations (the dashed and full lines are very similar for $M \geqslant 1 M_{\odot}$ ) while for a fixed mass a deviation is observed in the radius; deviation which increases as expected with decreasing mass. We consider that this comparison is representative of the systematic error which occurs for all functionals due to the limitations of the model. We have checked that this mass-dependent error bar is always smaller than the size of the symbols and width of the lines of all the figures of the present paper.

\section{RESULTS}

In the present section we discuss the uncertainties on the determination of the radius and the crust thickness of a star associated with the models presented in the previous section. In the next subsection we will also discuss how the radius and the crust thickness of NS are related with two properties at saturation: the incompressibility and the symmetry energy slope. We will then proceed to impose a set of terrestrial constraints and select the models that satisfy all constraints, or miss at most two by less than $10 \%$, and will discuss how the uncertainties on the determination of the radius and the crust thickness of a star previously obtained are affected. The final subsection focuses on the DUrca process and the possible constraint that could be put on $L$, the NS radius or the EOS thanks to the astrophysical observations of thermal states of NS.

\section{A. Radius and crust thickness}

In Fig. 9, the radii of $1.0 M_{\odot}, 1.4 M_{\odot}$, and $1.8 M_{\odot}$ NS for a purely nucleonic core are plotted as a function of the slope $L$ and the incompressibility $K$. We can see that the radii for the various EOS differ at most by $2.8,3.0$ and $3.7 \mathrm{~km}$ for masses of $1.0 M_{\odot}, 1.4 M_{\odot}$, and $1.8 M_{\odot}$, respectively. The uncertainty on the radius is connected with the nuclear properties of the EOS of the models used [88]. In the next subsection we will restrict ourselves to the models that also satisfy other constraints both from experiments and from theoretical calculations of pure neutron matter and will discuss how much this uncertainty changes.

We can also see from Fig. 9 that the radius appears well correlated with the slope of the symmetry energy $L$, especially for low mass stars. This correlation is still present for the more massive stars but the dispersion increases with the mass, as expected. Indeed, in [89] the authors have shown that the radius of low mass stars is well correlated with the neutron skin thickness of ${ }^{208} \mathrm{~Pb}$. On the other hand, it has been 
TABLE IV. Nuclear and astrophysical properties of considered Skyrme functionals. Energy per nucleon $\left(E_{\mathrm{s}}\right)$, compression modulus $(K)$, symmetry energy $(J)$, slope $(L)$ and incompressibility $\left(K_{\text {sym }}\right)$ of the symmetry energy at the saturation point of uniform symmetric nuclear matter of density $n_{\mathrm{s}} . M_{\max }^{\mathrm{noY}}$ is the maximum NS mass and $v_{\text {sound }}^{2}\left(2 M_{\odot}\right)$ is the square of the sound speed at a density equal to the central density of the $2 M_{\odot}$ NS. $n_{\mathrm{DU}}$ and $\mathrm{M}_{\mathrm{DU}}$ are respectively the density and mass threshold above which the nucleonic DUrca is switched on.

\begin{tabular}{|c|c|c|c|c|c|c|c|c|c|c|c|}
\hline Functional & $\begin{array}{c}n_{\mathrm{s}} \\
\left(\mathrm{fm}^{-3}\right)\end{array}$ & $\begin{array}{c}E_{\mathrm{s}} \\
(\mathrm{MeV})\end{array}$ & $\begin{array}{c}K \\
(\mathrm{MeV})\end{array}$ & $\begin{array}{c}J \\
(\mathrm{MeV})\end{array}$ & $\begin{array}{c}L \\
(\mathrm{MeV})\end{array}$ & $\begin{array}{c}K_{\text {sym }} \\
(\mathrm{MeV})\end{array}$ & $\begin{array}{l}M_{\max }^{\text {noY }} \\
\left(M_{\odot}\right)\end{array}$ & $\begin{array}{c}v_{\text {sound }}^{2}\left(2 M_{\odot}\right) \\
\left(\mathrm{c}^{2}\right)\end{array}$ & $\begin{array}{c}n_{\mathrm{DU}} \\
\left(\mathrm{fm}^{-3}\right)\end{array}$ & $\begin{array}{l}M_{\mathrm{DU}} \\
\left(M_{\odot}\right)\end{array}$ & Ref. \\
\hline Ska & 0.155 & -15.99 & 263.16 & 32.91 & 74.62 & -78.46 & 2.22 & 0.61 & 0.37 & 1.23 & [73] \\
\hline SkI2 & 0.1575 & -15.77 & 241.0 & 33.4 & 104.3 & 70.7 & 2.17 & 0.56 & 0.26 & 0.92 & [74] \\
\hline SkI3 & 0.1577 & -15.98 & 258.2 & 34.83 & 100.5 & 73.0 & 2.25 & 0.54 & 0.26 & 0.92 & [74] \\
\hline SkI4 & 0.160 & -15.95 & 247.95 & 29.50 & 60.39 & -40.56 & 2.18 & 0.64 & 0.52 & 1.64 & [74] \\
\hline SkI6 & 0.159 & -15.89 & 248.17 & 29.90 & 59.24 & -46.77 & 2.20 & 0.62 & 0.51 & 1.66 & [75] \\
\hline SLy2 & 0.161 & -15.99 & 229.92 & 32.00 & 47.46 & -115.13 & 2.06 & 0.78 & & & [76] \\
\hline SLy230a & 0.160 & -15.99 & 229.90 & 31.99 & 44.30 & -98.3 & 2.11 & 0.72 & 0.82 & 2.00 & [77] \\
\hline SLy4 & 0.159 & -15.97 & 230.0 & 32.04 & 46.00 & -119.8 & 2.06 & 0.79 & & & [15] \\
\hline SkMP & 0.157 & -15.56 & 230.87 & 29.89 & 70.31 & -49.82 & 2.11 & 0.66 & 0.43 & 1.32 & [78] \\
\hline $\mathrm{SkOp}$ & 0.160 & -15.75 & 222.36 & 31.95 & 68.94 & -78.82 & 1.98 & 0.55 & 0.58 & 1.53 & [79] \\
\hline BSk20 & 0.1596 & -16.080 & 241.4 & 30.0 & 37.4 & -136.5 & 2.17 & 0.77 & & & [83] \\
\hline BSk21 & 0.1582 & -16.053 & 245.8 & 30.0 & 46.6 & -37.2 & 2.29 & 0.60 & 0.45 & 1.60 & [83] \\
\hline BSk22 & 0.1578 & -16.088 & 245.9 & 32.0 & 68.5 & 13.0 & 2.27 & 0.56 & 0.33 & 1.15 & [67] \\
\hline BSk23 & 0.1578 & -16.068 & 245.7 & 31.0 & 57.8 & -11.3 & 2.28 & 0.58 & 0.38 & 1.34 & [67] \\
\hline BSk24 & 0.1578 & -16.048 & 245.5 & 30.0 & 46.4 & -37.6 & 2.29 & 0.60 & 0.45 & 1.60 & [67] \\
\hline BSk25 & 0.1587 & -16.032 & 236.0 & 29.0 & 36.9 & -28.5 & 2.23 & 0.58 & 0.47 & 1.63 & [67] \\
\hline BSk26 & 0.1589 & -16.064 & 240.8 & 30.0 & 37.5 & -135.6 & 2.18 & 0.76 & & & [67] \\
\hline SLy9 & 0.151 & -15.80 & 229.84 & 31.98 & 54.86 & -81.42 & 2.16 & 0.65 & 0.56 & 1.72 & [76] \\
\hline
\end{tabular}

discussed in $[40,90,91]$ that the neutron skin thickness is very sensitive to the density dependence of the nuclear symmetry energy and, in particular, to the slope parameter $L$ at the normal nuclear saturation density. The correlation obtained in [89] corresponds, therefore, to a correlation between the star radius and the slope $L$. The authors take a set of four different models and within each span a wide range of neutron skin thicknesses by changing the density dependence of the symmetry energy. The correlation between the star radius and the neutron skin thickness of ${ }^{208} \mathrm{~Pb}$ is particularly strong for stars with masses $0.5 M_{\odot}$ and $0.75 M_{\odot}$. For $M=1.4 M_{\odot}$, although a clear correlation is still present, the spread of the distribution is wider showing a larger model dependence. The behavior was attributed to the stellar matter densities that were being explored within each type of star: for low mass the main contribution comes from densities close to the saturation density where all models behave similarly because most of them are fitted to finite nucleus properties. The properties of stars with larger masses are also determined by the high density EOS, corresponding to a range of densities where the higher order coefficients in the density expansion of the energy functional play an increasing role.

Looking now at the radius as a function of the incompressibility, a linear correlation is also observed as indicated by the nonzero value of the correlation coefficient. However, the spread of the data for the three masses considered is considerably larger than when considering correlations between $L$ and the radii. This can be quantified by looking at the result of a fit, using a linear regression, of the radius $R$ for different masses, with a linear function $a x+b$, where $x=L$ or $K$. The result of the fit, including the error bar on the two fitting parameters, is represented in Fig. 9 as a shaded area. In the case of $R(L)$ (upper panel), a well defined linear behavior can be extracted, even if the importance of higher order terms in the density expansion [92] can be inferred by the larger dispersion at high mass. In contrast, the error in the $b$ parameter is so large that no relevant information on $K$ can be extracted from the radius. This indicates that, as far as isoscalar properties are concerned, the influence of higher order terms cannot be neglected. An analytic parametrization for radii of NS with different masses in terms of properties of symmetric saturated matter was first discussed in [88] and a quite complex dependence on $K$, skewness parameter $K^{\prime}=27 n_{\mathrm{s}}^{3}\left(\partial^{3} E_{\mathrm{NM}} / \partial n^{3}\right)_{n_{\mathrm{s}}, \delta=0}$, and $L$ was highlighted.

The crust thickness for the RMF models is plotted as a function of the star mass in Fig. 10. We do not show results for the Skyrme parametrizations because the method used to describe the crust in these models does not allow for a precise calculation of the crust-core transition density, as explained before. For the models represented in Fig. 10, no correlations were found between the crust thickness and the slope $L$ or the incompressibility $K$ for stars with masses $1.0 M_{\odot}, 1.4 M_{\odot}$, and $1.8 M_{\odot}$. Excluding the DDH $\delta$ model that predicts the smallest thickness, we have obtained $1.6<l^{\mathrm{cr}}<2.1 \mathrm{~km}$ for a star with 

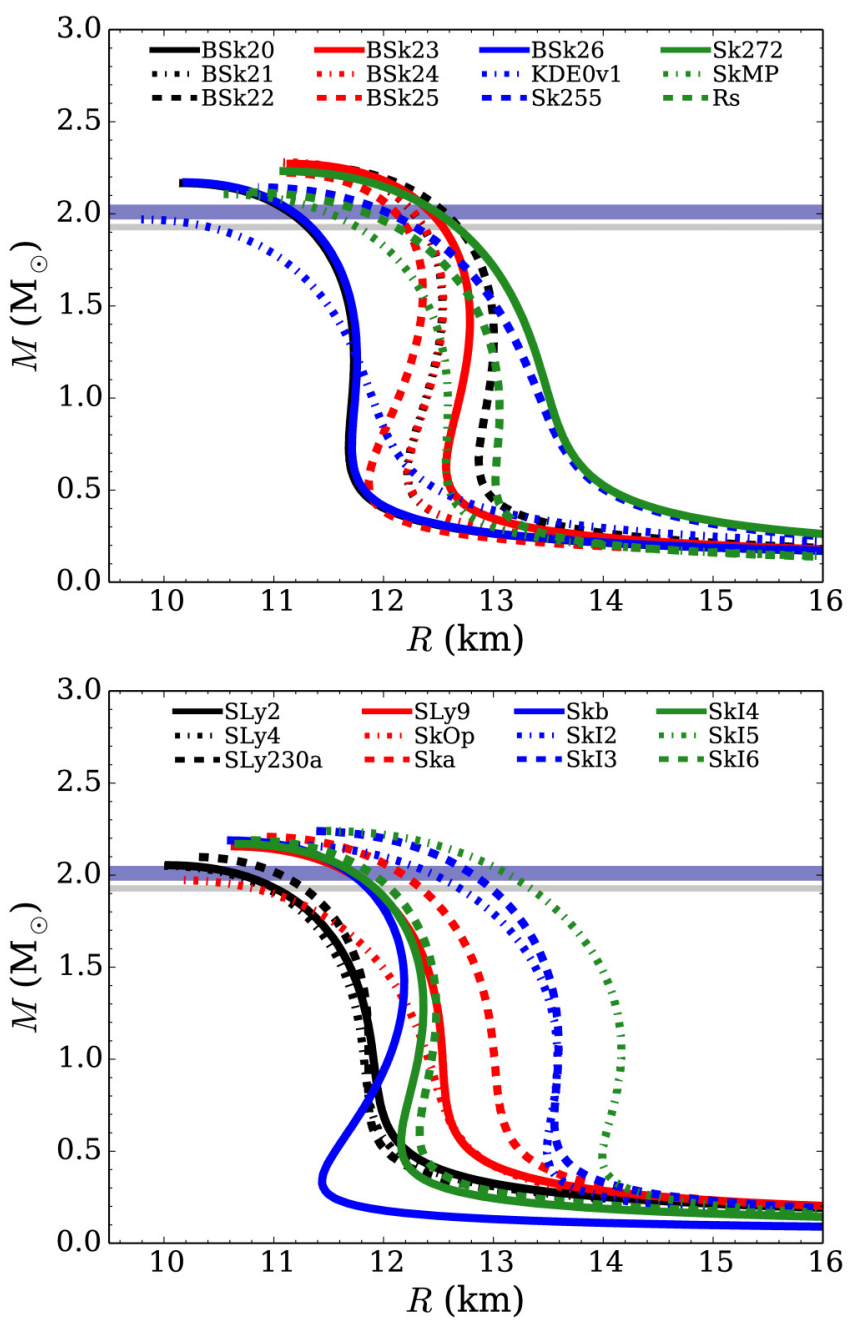

FIG. 7. Mass-radius relations for Skyrme models. As in Fig. 5, the horizontal lines indicate the constraints set by the pulsars PSR J0348+0432 and PSR J1614-2230.

$M=1.0 M_{\odot}, 1.1<l^{\text {cr }}<1.5 \mathrm{~km}$ for $1.4 M_{\odot}$ and $0.7<l^{\text {cr }}<$ $1.1 \mathrm{~km}$ for $1.8 M_{\odot}$. For stars with masses $1.0 M_{\odot}$ and $1.4 M_{\odot}$ the upper limits of the crust thickness are more than $30 \%$ larger than the lower limits. This difference rises to $50 \%$ for the $1.8 M_{\odot}$ star.

\section{B. Comparison with nuclear constraints}

So far, two constraints were imposed on the various EOS discussed in Sec. IV: the causality and ability to reach the $2 M_{\odot}$ mass limit. There are, however, several nuclear constraints that have been obtained from experiment or microscopic calculations during the last decade and which set much stronger conditions on the models. In this subsection we impose in addition the following set of constraints reviewed in [95-98]:

(1) Constraint on the energy per particle of neutron matter as a function of the density $n$ from the calculations of [94] and of [93] (see Fig. 11 and 12). From [94] we consider the limiting cases corresponding to the model

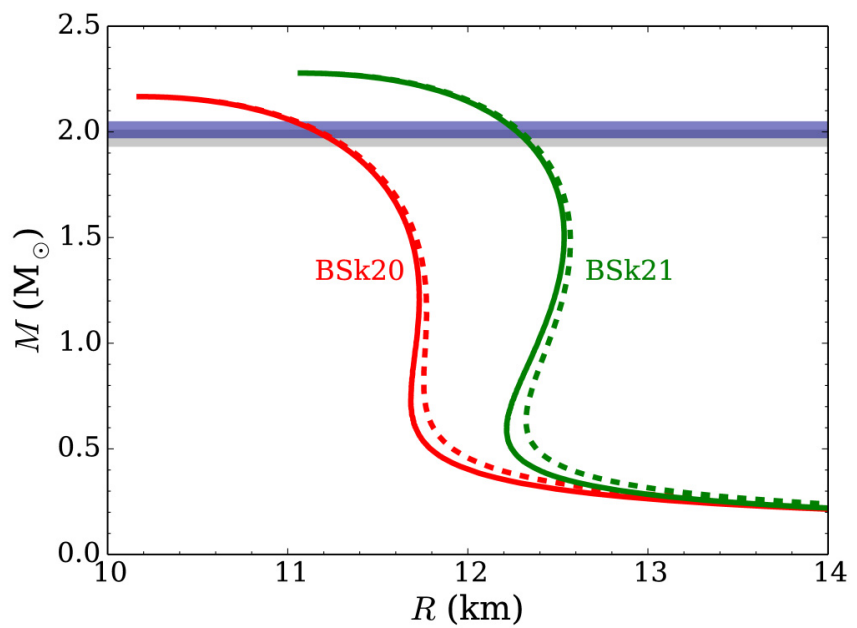

FIG. 8. Mass as a function of the radius for the BSk20 and BSk21 functionals. Full lines: full microscopic HFB calculation from [84]. Dashed lines: our model for the unified EOS. As in Fig. 5, the horizontal lines indicate the constraints set by the pulsars PSR J0348+0432 and PSR J1614-2230.

using a two-nucleon $(2 \mathrm{~N})$ force without $3 \mathrm{~N}$ interaction and to the one with a $2 \mathrm{~N}$ force and the Urbana IX $3 \mathrm{~N}$ interaction which approximately cover the present uncertainty range of ab initio calculations,

(2) Constraint on the incompressibility of infinite nuclear matter at saturation. Direct fits of ISGMR and IVGMR experimental data within a macroscopic approach based on the liquid drop model can lead to very different values for the $K$ parameter [99-101], but selfconsistent microscopic calculations in the last decade have lead to a reliable constraint $K=230 \pm 40 \mathrm{MeV}$ [102,103]. The quoted uncertainty include both the model dependence due to the use of relativistic or nonrelativistic functionals [102], and the uncertainty in the extrapolations from the average nuclear density to the saturation density [103].

(3) Constraints in the $J-L$ plane as compiled in $[95,97]$ and plotted in Fig. 13:

(a) from neutron skin thickness of ${ }^{208} \mathrm{~Pb}$ [104],

(b) from heavy ion collisions (HIC) [105],

(c) from electric dipole polarizability $\alpha_{\mathrm{D}}[97,106]$,

(d) from giant dipole resonance (GDR) of ${ }^{208} \mathrm{~Pb}$ [107],

(e) from measured nuclear masses [108],

(f) from isobaric analog states (IAS) [109].

In Table $\mathrm{V}$, all our models are confronted with this set of constraints: $\mathrm{Y}$ or $\mathrm{N}$ indicate whether the constraint is satisfied or not, respectively. For the neutron energy per particle from microscopic calculations we have also considered a less restrictive constraint increasing by $10 \%$ the uncertainty interval. The constraint on the incompressibility is taken from [103]. However, in [110] it was discussed that the uncertainty in the incompressibility is related to the lack of knowledge of the skewness. Taking both Skyrme interactions and RMF models, the uncertainty in the skewness is larger than $\pm 400 \mathrm{MeV}$, so that the uncertainty of $17 \%$ (corresponding 

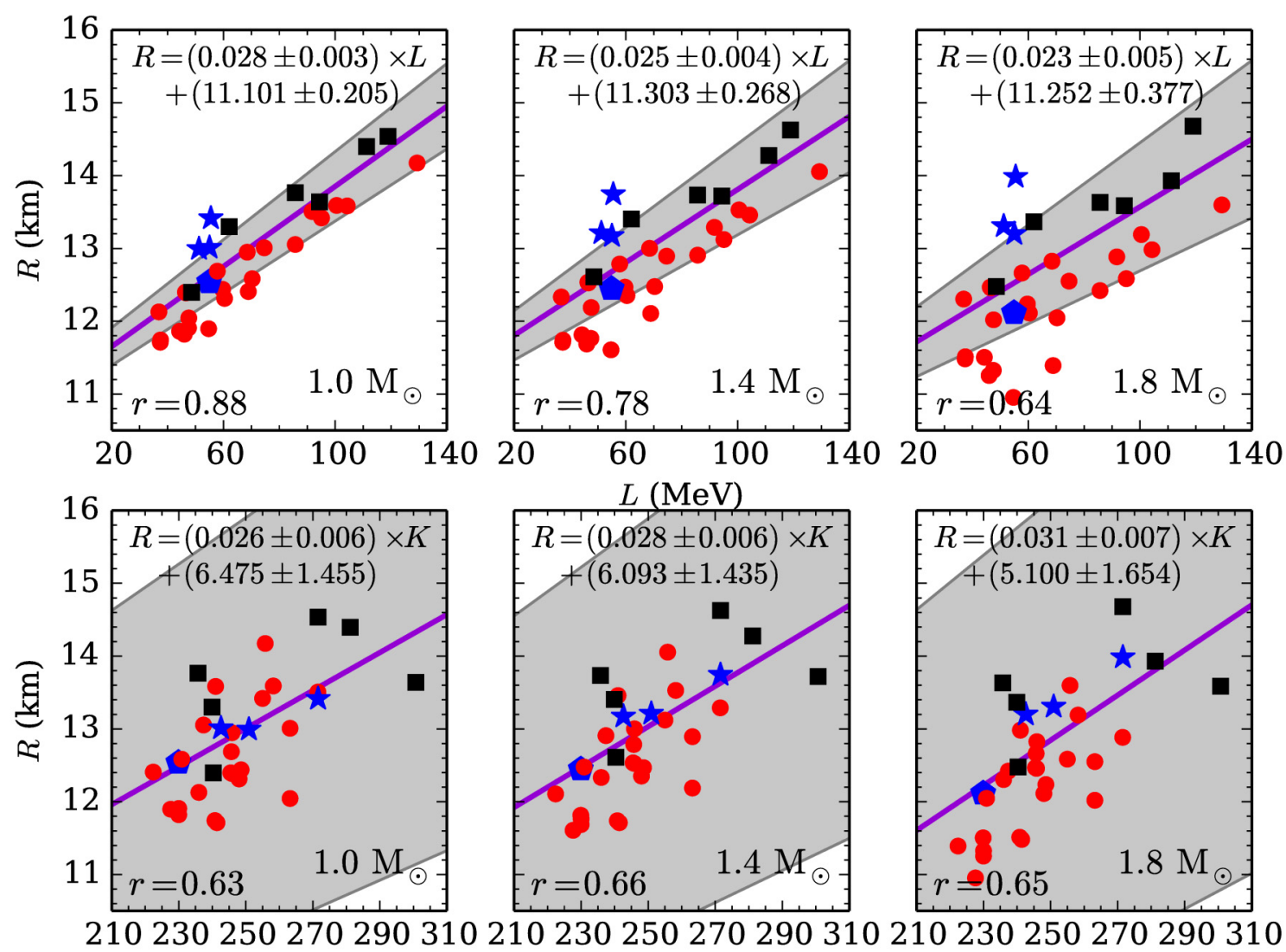

$K(\mathrm{MeV})$

FIG. 9. Radii of purely nucleonic NS as a function of the symmetry energy slope $L$ (upper plots) and the incompressibility of symmetric matter $K$ (lower plots) for different masses $\left(1.0 M_{\odot}, 1.4 M_{\odot}\right.$, and $1.8 M_{\odot}$ from left to right). The red dots indicate Skyrme models, the black ones RMF models. The blue symbols (stars for RMF and a pentagon for SLy9) correspond to models which are at the intersection of all nuclear constraints in the $L-J$ plane; see Fig. 13. The shaded areas and the solid violet line indicate the result of a linear regression with and without, respectively, taking into account the error bars in the fitted parameters. The correlation coefficient $r$ is indicated in each plot.

to $40 \mathrm{MeV}$ ) obtained for the incompressibility in [103] may be underestimated taking into account that in their analysis only three RMF models were considered. We therefore relax this constraint and consider that NL3, NL3 $\omega \rho$, and Sk272 also satisfy the constraint corresponding to increasing the uncertainty from $17 \%$ to $18 \%$. This is indicated by the * symbol in Table $\mathrm{V}$.

Only one model satisfies all the constraints: DDME2. Increasing the uncertainty interval of the energy per particle of neutron matter from the calculations of [93] and [94], three more models can be selected: DD2, NL3 $\omega \rho$, and SLy9. In Fig. 9 we present the three selected RMF models with a blue star and the one Skyrme interaction with a blue pentagon.

In Fig. 14 the mass-radius curves of the selected models are shown for EOS of purely nucleonic and hyperonic matter (if available). Although they all have a very similar $L-$ three of them have $L \sim 55 \mathrm{MeV}$ and for the last one $L=51.2$ $\mathrm{MeV}$ - the radius uncertainty of a $1.4 M_{\odot}$ star spanned by these models is $\Delta R_{1.4}=1.30 \mathrm{~km}$ defined by the difference between $R_{1.4}(\mathrm{SLy} 9)=12.45 \mathrm{~km}$ and $R_{1.4}(\mathrm{NL} 3 \omega \rho)=13.75 \mathrm{~km}$. This uncertainty reduces to $0.88 \mathrm{~km}$ for $1.0 M_{\odot}$ stars and increases to $2.34 \mathrm{~km}$ for $2.0 M_{\odot}$ stars. This radius interval is $\sim 1 / 3$ of the one that was obtained in Sec. IV for $1.0 M_{\odot}$ stars and $\sim 1 / 2$ for $2.0 M_{\odot}$ stars. The fact that the range of possible radii is larger for the more massive stars reflects the fact that the high density range of the EOS is less well constrained than the one close to and below saturation density.

One property that is very different for all the four models, represented by a blue mark, is the incompressibility $K$; see Fig. 9, bottom panels. The radius of the star is also to some extent correlated with the incompressibility but, as discussed above, the uncertainty of the linear correlation is too large to provide a further constraint.

The same set of experimental constraints employed in this work has been previously used in [97] with the same aim of addressing the relation between uncertainties on the nuclear EOS and NS radii. Using analytic equations between NS radii and pressure of beta-equilibrated matter, which in turn depends on $L$ and $K$, the interval of $12.1 \pm 1.1 \mathrm{~km}$ was proposed, within $90 \%$ confidence, for the radius of $1.4 M_{\odot}$.

Finally, let us focus on the crust properties of the selected RMF models, which have been plotted together in the upper panel of Fig. 10. The dispersion observed in the crust thickness becomes narrower, corresponding to $\sim 250 \mathrm{~m}$, 

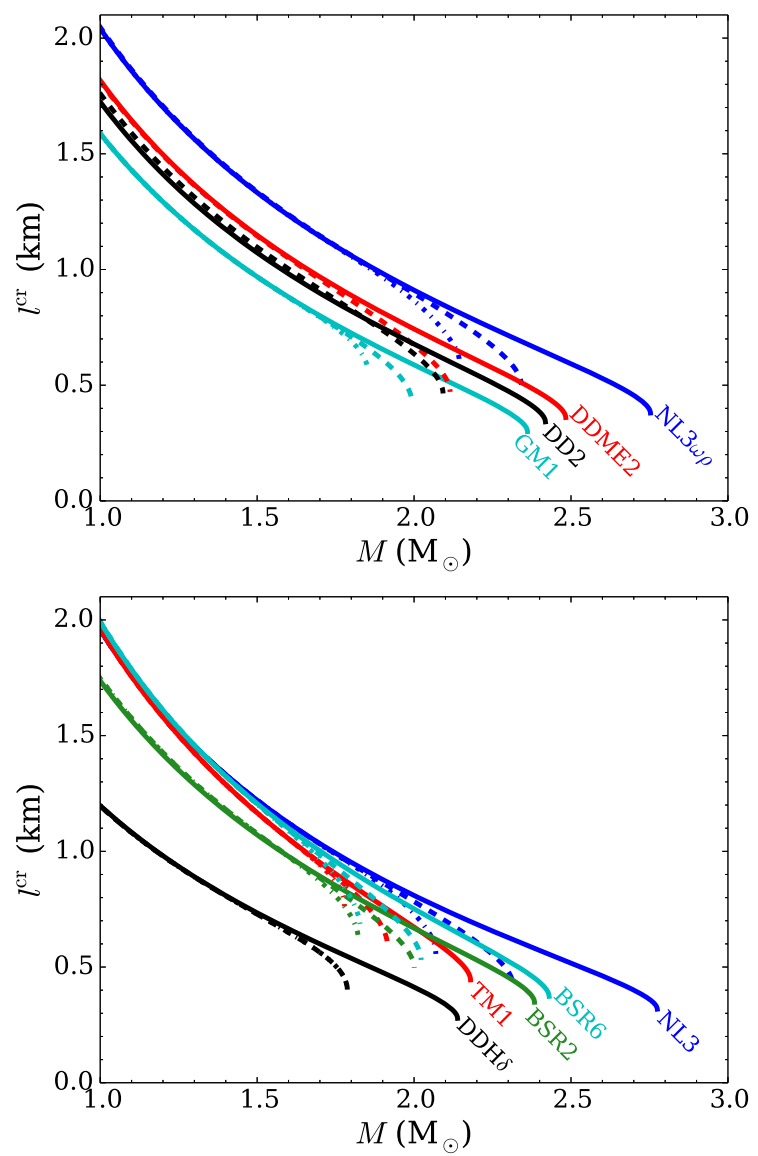

FIG. 10. Mass vs crust thickness relation for the various RMF models: noY, Y, and Yss. Line styles correspond to the ones used in Fig. 5.

which represents $\sim 30 \%$ of the range obtained for all RMF models.

We can conclude that the present knowledge of $L$ and $K$ allows determining the NS radius within $1-2 \mathrm{~km}$. This residual uncertainty appears to be essentially due to the lacking information on higher order terms, meaning that an increasing precision in the constraints for $L$ and $K$ is going to improve this prediction only marginally.

This underlines the importance of independent constraints. One possibility would be to get information on higher order coefficients (skewness, symmetry incompressibility, etc.) from high density laboratory observables.

\section{DUrca processes}

An interesting way to constrain the EOS could be to exploit independent astrophysical data, notably from the luminosity curves of the accreting NS and their interplay with the possible occurrence of the DUrca process. This connection is explained in the following.

After their birth in supernova explosions, NS are efficiently cooled down by neutrino emission during $\sim 10^{5}-10^{6}$ years (see [111] and references therein). The simplest possible and most powerful neutrino process is the so-called nucleonic electron
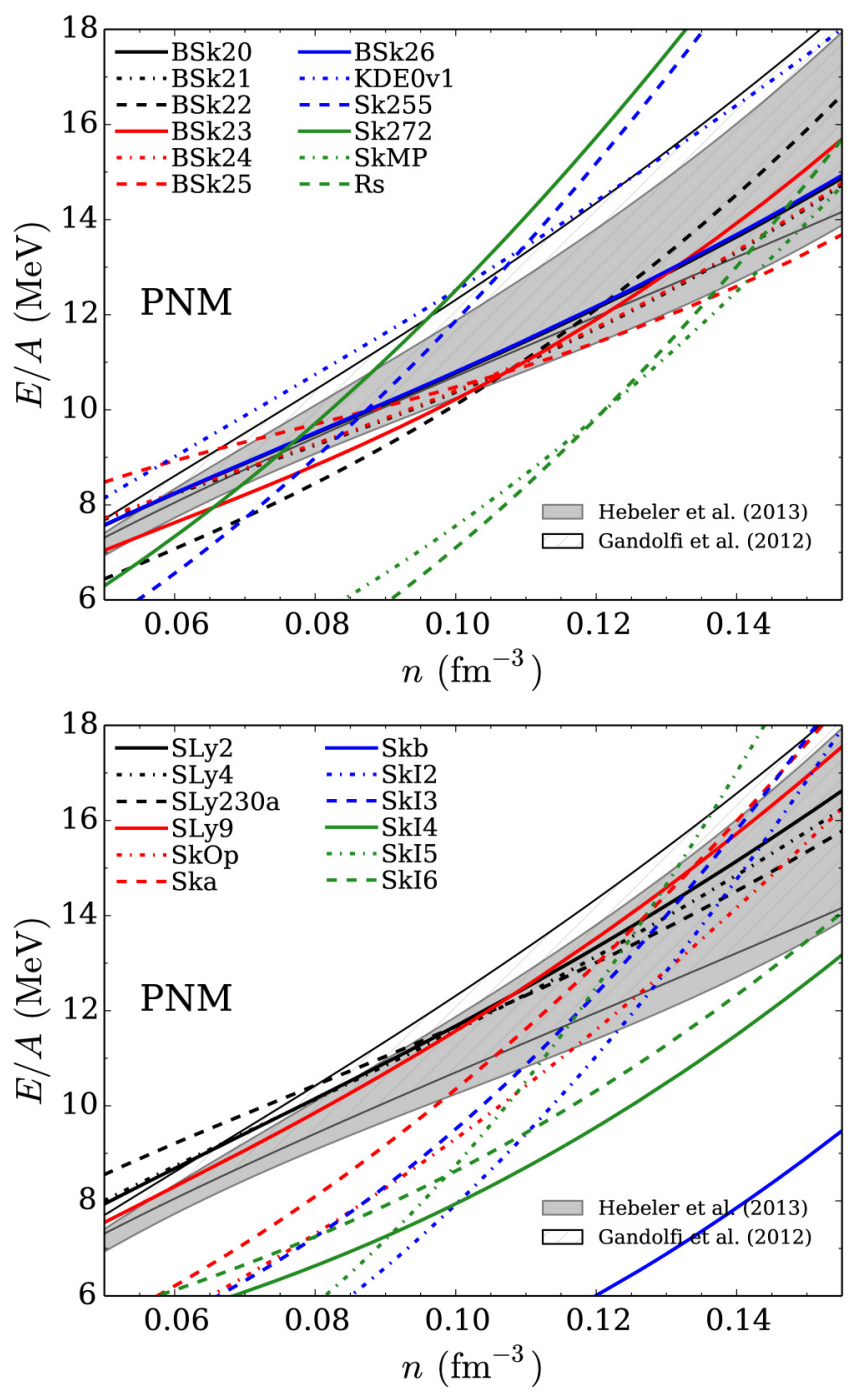

FIG. 11. Energy per particle of neutron matter as a function of density $n$ for Skyrme models and constraints by [93] and [94].

DUrca process [112]:

$$
n \rightarrow p+e^{-}+\bar{v}_{e} \text { and } p+e^{-} \rightarrow n+v_{e},
$$

which corresponds to the neutron $\beta$ decay followed by the electron capture on the proton. Momentum conservation has to be satisfied for this process to operate which translates into the so-called triangle inequalities:

$$
p_{\mathrm{F} n} \leqslant p_{\mathrm{F} p}+p_{\mathrm{F} e},
$$

where $p_{\mathrm{F} i}$ is the Fermi momentum of a species $i$. Such inequalities impose a minimum proton fraction $Y_{p}^{\min }$ for the nucleonic DUrca process to occur [113]:

$$
Y_{p}^{\min }=\frac{1}{1+\left(1+x_{e}^{1 / 3}\right)^{3}},
$$

with $x_{e}=n_{e} /\left(n_{e}+n_{\mu}\right)$. The absence of muons corresponds to $x_{e}=1$ and $Y_{p}^{\min }=1 / 9$ while their inclusion results in an increase of the value of $Y_{p}^{\mathrm{min}}$. This minimum proton fraction translates into a threshold density $n_{\mathrm{DU}}$ and mass $M_{\mathrm{DU}}$ above 


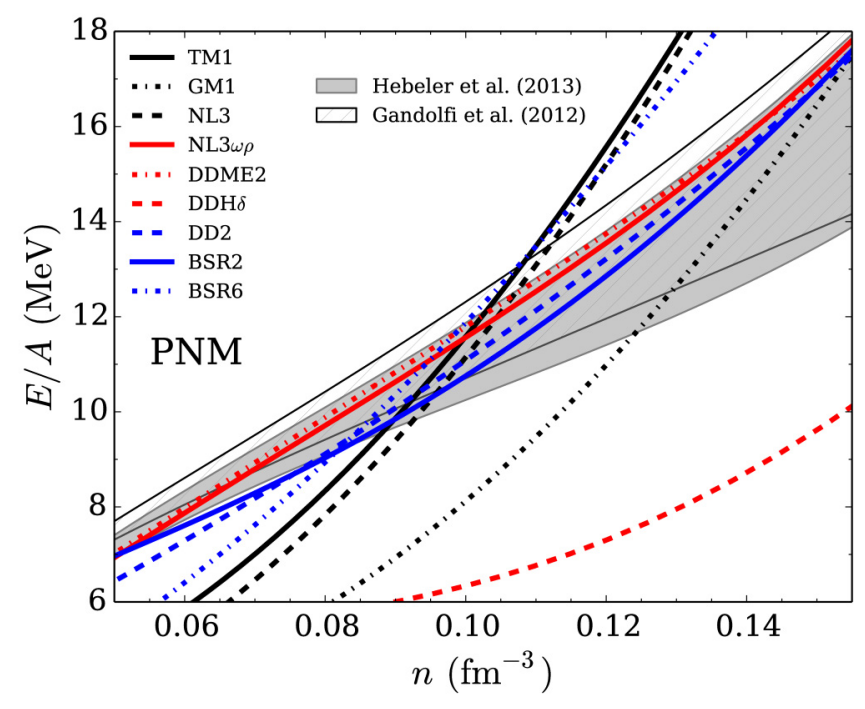

FIG. 12. Energy per particle of neutron matter as a function of density $n$ for RMF models and constraints by [93] and [94].

which the DUrca process is active. A process similar to the one in Eq. (26) but involving muons instead of electrons may also operate; its threshold density is then slightly larger than for the electron DUrca process.

Mass and density thresholds for the nucleonic DUrca process in purely nucleonic cores are given in Tables II and IV for RMF and Skyrme models, respectively. For some EOS, the density threshold above which the DUrca process operates is larger than the central density of the maximum-mass configuration. In other words, for such EOS the DUrca process is turned off for all possible masses and does not operate for any NS configuration. In Fig. 15 the density threshold above

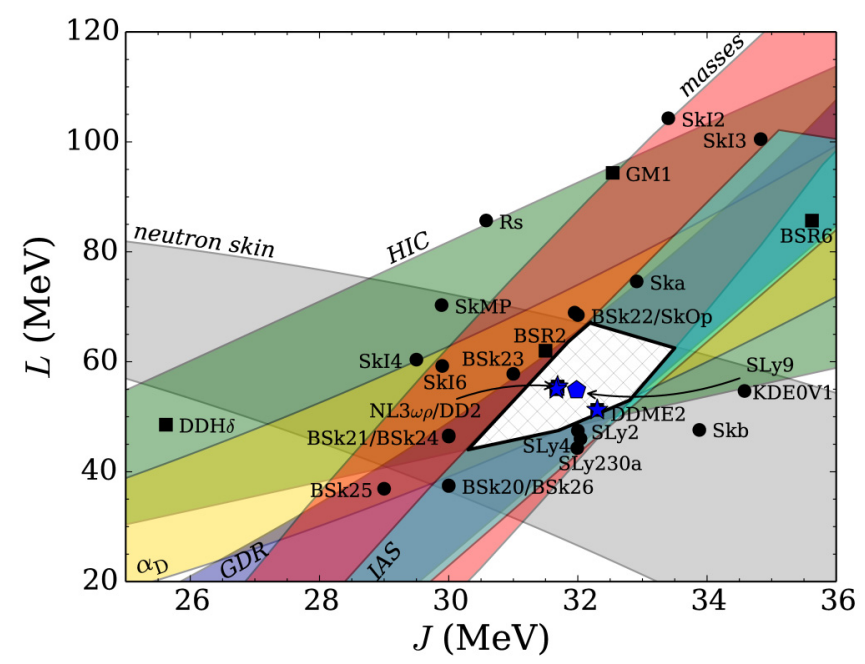

FIG. 13. $L$ and $J$ parameters of all our EOS compared to various nuclear constraints (see text for details). The white crossed region corresponds to the intersection of all constraints. EOS fulfilling all constraints are indicated by blue symbols: a pentagon for the unique Skyrme model and a star for the three RMF ones. which the DUrca process operates in a purely nucleonic NS and the mass of the star with the corresponding central density are plotted against the slope of the symmetry energy. It reveals the possible existence of two distinct regions defined by a threshold on $L: L^{\mathrm{DU}} \simeq 70 \mathrm{MeV}$. Every nonhyperonic EOS with $L \gtrsim L^{\mathrm{DU}}$ has the DUrca process operating in NS above a mass $M<1.5 M_{\odot}$. This is not the case for EOS with $L \lesssim L^{\mathrm{DU}}$ as for some EOS the DUrca does not operate at any NS mass and, for other EOS, it does for masses either above $2 M_{\odot}$ or close to $1.5 M_{\odot}$. Regarding EOS that fulfill all constraints, two patterns are to be noted. For SLy9 the DUrca process is possible for masses larger than $1.72 M_{\odot}$. For the three RMF models, in spite of their similar values of $J$ and $L$, only the one with non-density-dependent coupling constants, NL3 $\omega \rho$, allows for the DUrca process, although at high density and masses above $2.5 M_{\odot}$.

On the one hand, the thermal state of SAX J1808.4-3658, the coldest observed transiently accreting NS, can be explained as shown in [21] by a very large neutrino emission in the core of the NS that only the very efficient DUrca process can explain. Interestingly the region where all nuclear constraints in the $L-J$ plane overlap, as plotted in Fig. 13, corresponds to values of $L$ that are strictly smaller than the same $L^{\mathrm{DU}}$ below which the DUrca process does not necessarily operate in massive purely nucleonic NS. Therefore reconciling the nuclear constraints on $L$ and $J$ with the astrophysical one that the DUrca process operates in NS might be challenging, as shown by the fact that out of four EOS fulfilling our set of constraints only one (SLy9) allows for the DUrca process below $2 M_{\odot}$. In other words astrophysical observations of NS with a low luminosity might constrain the value of $L$ and consequently the radius as discussed in Sec. V A. On the other hand, population synthesis of isolated NS imposes that the DUrca process does not occur in NS with masses $1.0 M_{\odot}-1.5 M_{\odot}$ [114], a constraint that only a small number of EOS satisfy with $L \lesssim 70 \mathrm{MeV}$, as shown in Fig. 15, unless a strong proton superfluidity occurs in the core of low mass stars [21]. In particular, among the EOS consistent with our set of nuclear constraints, SLy9, NL3 $\omega \rho$ for a purely nucleonic interior and an hyperonic one, and DD2 with hyperons do not have any DUrca process below $1.5 M_{\odot}$.

DUrca processes similar to the nucleonic ones can also occur in hyperonic NS [115]. Examples relevant for our EOS are

$$
\begin{gathered}
\Lambda \rightarrow p+l+\bar{v}_{l} \text { and } p+l \rightarrow \Lambda+v_{l}, \\
\Xi^{-} \rightarrow \Lambda+l+\bar{v}_{l} \text { and } \Lambda+l \rightarrow \Xi^{-}+v_{l}, \\
\Xi^{-} \rightarrow \Xi^{0}+l+\bar{v}_{l} \text { and } \Xi^{0}+l \rightarrow \Xi^{-}+v_{l} .
\end{gathered}
$$

The DUrca process involving a given hyperon turns on at a density very close to the onset density of this specific hyperon (with the condition that all other species involved in the process are also present). These density thresholds, or equivalently mass thresholds, for our RMF hyperonic EOS are given in Table III together with the same quantity for the nucleonic DUrca process. In the presence of hyperons the onset of the 
TABLE V. Confrontation of the EOS with the various constraints. For each model the symbols $\mathrm{Y}$ and $\mathrm{N}$ indicate whether a given constraint is fulfilled or not, respectively. For the constraints from [93] and [94] on the energy per particle of neutron matter, the interval of density over

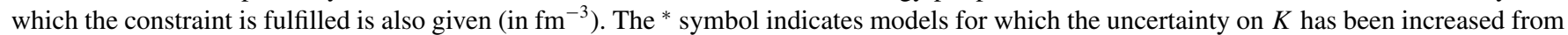
$17 \%$ to $18 \%$; see text for details. In boldface we indicate the EOS that satisfy all constraints and that are also represented by blue marks in Fig. 9.

\begin{tabular}{|c|c|c|c|c|c|c|c|c|c|c|c|}
\hline Model & Hebeler & Hebeler $+10 \%$ & Gandolfi & Gandolfi $+10 \%$ & $K$ & Neutron skin & HIC & $\alpha_{\mathrm{D}}$ & GDR & masses & IAS \\
\hline NL3 & N 0.093-0.108 & $\mathrm{N} 0.084-0.120$ & $\mathrm{~N} 0.096-0.112$ & N $0.087-0.125$ & $\mathrm{Y}^{*}$ & $\mathrm{~N}$ & $\mathrm{~N}$ & $\mathrm{~N}$ & $\mathrm{~N}$ & $\mathrm{~N}$ & $\mathrm{~N}$ \\
\hline NL3 $\omega \rho$ & $\mathrm{N} 0.051-0.155$ & Y $0.050-0.155$ & $\mathrm{~N} 0.068-0.155$ & Y $0.050-0.155$ & $\mathrm{Y}^{*}$ & $\mathrm{Y}$ & $\mathrm{Y}$ & $\mathrm{Y}$ & $\mathrm{Y}$ & $\mathrm{Y}$ & $\mathrm{Y}$ \\
\hline GM1 & $\mathrm{N} 0.124-0.155$ & $\mathrm{~N} 0.113-0.155$ & $\mathrm{~N} 0.130-0.155$ & N $0.118-0.155$ & $\mathrm{~N}$ & $\mathrm{~N}$ & $\mathrm{Y}$ & $\mathrm{N}$ & $\mathrm{N}$ & $\mathrm{N}$ & $\mathrm{N}$ \\
\hline TM1 & N 0.089-0.103 & $\mathrm{N} 0.078-0.116$ & N 0.092-0.109 & N 0.082-0.122 & $\mathrm{N}$ & $\mathrm{N}$ & $\mathrm{N}$ & $\mathrm{N}$ & $\mathrm{N}$ & $\mathrm{N}$ & $\mathrm{N}$ \\
\hline DDHd & N $0.155-0.155$ & N $0.155-0.155$ & N $0.155-0.155$ & N $0.155-0.155$ & $\mathrm{Y}$ & $\mathrm{N}$ & $\mathrm{Y}$ & $\mathrm{N}$ & $\mathrm{N}$ & $\mathrm{N}$ & $\mathrm{N}$ \\
\hline BSR2 & $\mathrm{N} 0.083-0.155$ & Y $0.050-0.155$ & N 0.099-0.155 & Y $0.050-0.155$ & $\mathrm{Y}$ & $\mathrm{Y}$ & $\mathrm{Y}$ & $\mathrm{Y}$ & $\mathrm{N}$ & $\mathrm{Y}$ & $\mathrm{N}$ \\
\hline BSR6 & N 0.082-0.101 & $\mathrm{N} 0.070-0.120$ & N 0.086-0.108 & N $0.074-0.131$ & $\mathrm{Y}$ & $\mathrm{N}$ & Y & $\mathrm{Y}$ & $\mathrm{Y}$ & $\mathrm{N}$ & $\mathrm{Y}$ \\
\hline Ska & N 0.099-0.140 & N $0.082-0.155$ & N 0.106-0.153 & N $0.088-0.155$ & $\mathrm{Y}$ & $\mathrm{N}$ & $\mathrm{Y}$ & $\mathrm{Y}$ & $\mathrm{N}$ & $\mathrm{Y}$ & $\mathrm{Y}$ \\
\hline Skb & $\mathrm{N} 0.155-0.155$ & N $0.155-0.155$ & N $0.155-0.155$ & N $0.155-0.155$ & $\mathrm{Y}$ & $\mathrm{Y}$ & $\mathrm{N}$ & $\mathrm{N}$ & $\mathrm{N}$ & $\mathrm{N}$ & $\mathrm{N}$ \\
\hline SkI2 & $\mathrm{N} 0.123-0.155$ & $\mathrm{~N} 0.113-0.155$ & N 0.128-0.155 & N $0.118-0.155$ & $\mathrm{Y}$ & $\mathrm{N}$ & $\mathrm{N}$ & $\mathrm{N}$ & $\mathrm{N}$ & $\mathrm{N}$ & $\mathrm{N}$ \\
\hline SkI3 & N $0.110-0.143$ & N $0.096-0.155$ & N 0.116-0.151 & N 0.102-0.155 & $\mathrm{Y}$ & $\mathrm{N}$ & $\mathrm{Y}$ & $\mathrm{N}$ & $\mathrm{N}$ & $\mathrm{Y}$ & $\mathrm{N}$ \\
\hline SLy230a & N 0.092-0.155 & N $0.061-0.155$ & N $0.081-0.155$ & N $0.053-0.155$ & $\mathrm{Y}$ & $\mathrm{Y}$ & $\mathrm{N}$ & $\mathrm{N}$ & $\mathrm{N}$ & $\mathrm{Y}$ & $\mathrm{Y}$ \\
\hline SLy4 & N $0.080-0.155$ & Y $0.050-0.155$ & N 0.066-0.155 & Y $0.050-0.155$ & $\mathrm{Y}$ & $\mathrm{Y}$ & $\mathrm{N}$ & $\mathrm{N}$ & $\mathrm{Y}$ & $\mathrm{Y}$ & $\mathrm{Y}$ \\
\hline SLy9 & N $0.057-0.155$ & Y $0.050-0.155$ & Y $0.050-0.155$ & Y $0.050-0.155$ & $\mathrm{Y}$ & Y & $\mathrm{Y}$ & $\mathrm{Y}$ & $\mathrm{Y}$ & $\mathrm{Y}$ & $\mathrm{Y}$ \\
\hline SkMP & $\mathrm{N} 0.143-0.155$ & N $0.127-0.155$ & N 0.149-0.155 & N $0.133-0.155$ & $\mathrm{Y}$ & $\mathrm{Y}$ & $\mathrm{Y}$ & $\mathrm{N}$ & $\mathrm{N}$ & $\mathrm{N}$ & $\mathrm{N}$ \\
\hline $\mathrm{SkOp}$ & $\mathrm{N} 0.117-0.155$ & N 0.099-0.155 & $\mathrm{N} 0.126-0.155$ & N 0.106-0.155 & $\mathrm{Y}$ & $\mathrm{N}$ & $\mathrm{Y}$ & $\mathrm{Y}$ & $\mathrm{N}$ & $\mathrm{Y}$ & $\mathrm{N}$ \\
\hline KDE0v1 & N $0.155-0.155$ & N $0.051-0.155$ & $\mathrm{~N} 0.125-0.155$ & Y $0.050-0.155$ & $\mathrm{Y}$ & $\mathrm{Y}$ & $\mathrm{N}$ & $\mathrm{N}$ & $\mathrm{N}$ & $\mathrm{N}$ & $\mathrm{N}$ \\
\hline Sk255 & N 0.082-0.101 & $\mathrm{N} 0.068-0.120$ & N 0.086-0.108 & N $0.073-0.130$ & $\mathrm{Y}$ & $\mathrm{N}$ & $\mathrm{N}$ & $\mathrm{N}$ & $\mathrm{N}$ & $\mathrm{N}$ & $\mathrm{N}$ \\
\hline Sk272 & N $0.070-0.089$ & $\mathrm{~N} 0.050-0.110$ & N $0.075-0.096$ & N $0.058-0.121$ & $\mathrm{Y}^{*}$ & $\mathrm{~N}$ & $\mathrm{~N}$ & $\mathrm{~N}$ & $\mathrm{~N}$ & $\mathrm{~N}$ & $\mathrm{~N}$ \\
\hline Rs & N 0.137-0.155 & $\mathrm{N} 0.125-0.155$ & N 0.142-0.155 & N 0.130-0.155 & $\mathrm{Y}$ & $\mathrm{N}$ & $\mathrm{N}$ & $\mathrm{N}$ & $\mathrm{N}$ & $\mathrm{N}$ & $\mathrm{N}$ \\
\hline BSk20 & N $0.056-0.155$ & Y $0.050-0.155$ & Y $0.050-0.155$ & Y $0.050-0.155$ & $\mathrm{Y}$ & $\mathrm{Y}$ & $\mathrm{N}$ & $\mathrm{N}$ & $\mathrm{Y}$ & $\mathrm{Y}$ & $\mathrm{Y}$ \\
\hline BSk21 & $\mathrm{N} 0.057-0.155$ & Y $0.050-0.155$ & $\mathrm{~N} 0.050-0.070$ & Y $0.050-0.155$ & $\mathrm{Y}$ & $\mathrm{Y}$ & $\mathrm{Y}$ & $\mathrm{Y}$ & $\mathrm{Y}$ & $\mathrm{Y}$ & $\mathrm{N}$ \\
\hline
\end{tabular}

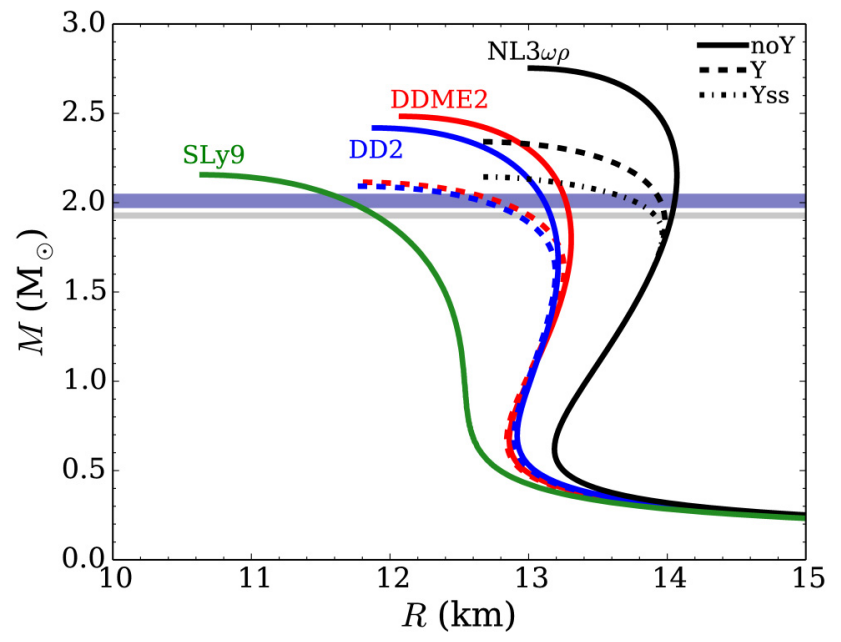

FIG. 14. Mass-radius relations for the models fulfilling all nuclear constraints. As in Fig. 5, the horizontal lines indicate the constraints set by the pulsars PSR J0348+0432 and PSR J1614-2230. nucleonic DUrca process occurs at

$$
\left(\frac{n_{p}}{n_{p}+n_{n}}\right)=\frac{1}{1+\left(1+x_{e}^{Y 1 / 3}\right)^{3}}, \quad x_{e}^{Y}=\frac{n_{e}}{n_{e}+n_{\mu}-n_{Y}^{c h}},
$$

where $n_{Y}^{c h}=-n_{\Sigma^{-}}+n_{\Sigma^{+}}-n_{\Xi^{-}}$.

The hyperonic DUrca processes have weaker emissivities than their nucleonic counterparts [115], but for some EOS they actually turn on at densities lower than the threshold for the nucleonic process. For the hyperonic version of our selected EOS-DD2, DDME2, and NL3 $\omega \rho$ - the mass thresholds are $1.52 M_{\odot}, 1.41 M_{\odot}$, and $1.58 M_{\odot}$, respectively. Nevertheless, a systematic study of the dependence of these thresholds on the poorly constrained hyperon properties and on the nuclear parameters (e.g., $L$ ) is beyond the scope of this paper and will be the subject of future works. It should be reminded that in the presence of hyperons the nucleonic DUrca process occurs at lower densities than expected in purely nucleonic stars due to the smaller neutron fraction, because hyperons contribute to both $\rho$ and charge density. However, the condition for the 


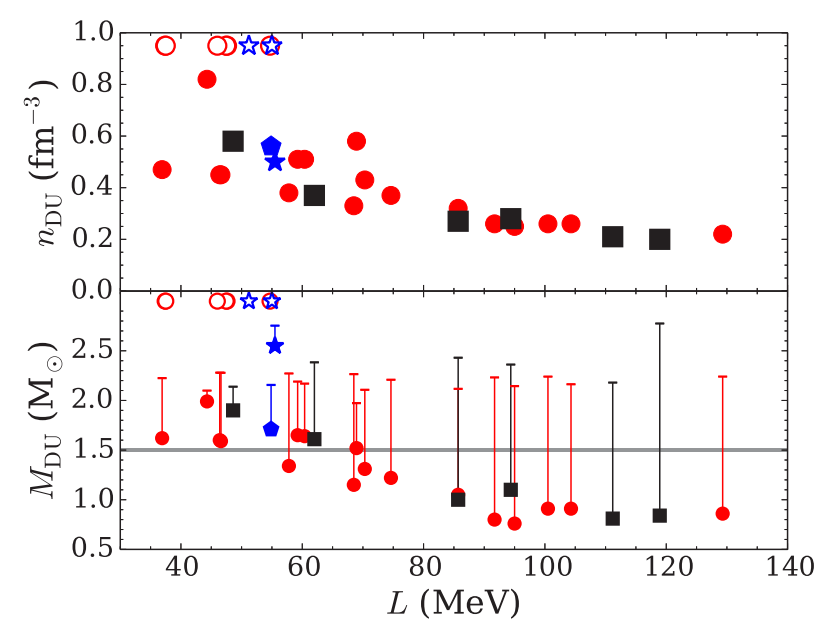

FIG. 15. Density threshold $n_{\text {DU }}$ (upper plot) and mass threshold $M_{\mathrm{DU}}$ (lower plot) for the nucleonic DUrca process to operate in purely nucleonic NS versus the slope of the symmetry energy $L$. The convention for colors and symbols is the same as in Fig. 9. Empty symbols next to the upper $x$ axis indicate EOS for which $n_{\mathrm{DU}}$ is larger than the central density of the most-massive NS. In the lower plot, the error bars indicate the mass range over which the DUrca process operates.

nucleon DUrca process is still given by the inequality (27), and a similar one with electrons replaced by muons. This could be an indication that it is necessary to take into account hyperons in order to reconcile an efficient DUrca process and an $L$ restricted to the interval allowed by terrestrial experiments.

\section{CONCLUSION}

The present study has two main objectives: (i) to illustrate the uncertainty that arises in the star radius determination when a non-unified EOS is used for the integration of the TOV equations and (ii) to quantify the same uncertainty taking a set of causal unified EOS that are consistent with the $2 M_{\odot}$ maximum-mass limit, with or without considering an extra set of constraints.

The unified EOS that are presented have been chosen among the nuclear RMF models and Skyrme interactions. In the latter case we have only considered models with causal EOS for densities at least as high as the central density of a $2 M_{\odot}$ star. We have also considered EOS with hyperonic degrees of freedom for all the chosen RMF models. Except for DDH $\delta$ and TM1, all the other hyperonic EOS could still describe a $2 M_{\odot}$ star when obtained using $\mathrm{SU}(6)$ symmetry to fix the vector meson coupling, experimental results to fix the scalar meson couplings, and, considering the mesons with hidden strangeness, including the $\phi$ meson and excluding the $\sigma^{*}$ meson.

The unified EOS were built using different approaches for the RMF and Skyrme models. For the RMF EOS we take the same outer crust EOS [24] for all the models, the inner crust is obtained within a Thomas Fermi calculation performed allowing for nonspherical clusters according to $[38,55]$, and the core is described by the homogeneous matter EOS. The EOS are not completely unified due to the outer crust EOS, since this EOS is mainly fixed by experimental measurements; the effect of this approximation is however very small. Considering the nonrelativistic unified EOS: at low density the nucleus $A$ and $Z$ numbers, as well as the volume $V_{\mathrm{WS}}$ of the Wigner-Seitz cell and the density of the free neutron component after drip, are variationally determined. The free neutrons are described with the same functional used for the calculation of the core EOS. Concerning the nucleus, it is modeled with a compressible liquid-drop model with parameters fitted from Hartree-Fock calculations employing the same Skyrme functional. Modifications of cluster energy functionals due to in-medium surface corrections, disregarded by the present modeling, will be addressed within the extended Thomas-Fermi approximation in a forthcoming paper. With the same occasion the correlations between the crust-core transition density, crust thickness, and the properties of uniform nuclear matter will be also discussed.

It was shown that for the non-unified EOS the crust-core matching may quite strongly affect the radius and crust thickness of the less massive stars. For our examples, depending on the matching procedure the difference in the radius and in crust thickness for a $1.0 M_{\odot}$ star can be as large as $\sim 1$ and $\sim 0.5 \mathrm{~km}$, respectively. This corresponds to relative differences as large as $\sim 4 \%$ for the radius and $30 \%$ for the crust thickness. The largest uncertainties occur when the density dependence of the symmetry energy is not the same in the crust and the core (i.e., different slopes $L$ characterize the two EOS). This uncertainty may be minimized if EOS for the crust and the core with similar saturation properties are considered, when a unified EOS is not available.

Taking the initial set of EOS we have shown that the spanned range of radii is $\sim 3$ and $\sim 4 \mathrm{~km}$ wide for $1.0 M_{\odot}$ and $2.0 M_{\odot}$ respectively. Imposing further constraints from experiment and theoretical calculations of neutron matter, these intervals for radii are reduced respectively, to $\sim 1$ and $2 \mathrm{~km}$. Although smaller, this uncertainty is still large and reflects mostly our ignorance on the high density EOS, or equivalently on the higher order terms of the density expansion of the energy functional. Additional uncertainties arise when the hyperon degrees of freedom are available. If hyperons are considered it is still possible to get $2 M_{\odot}$ stars, meaning that they cannot be simply neglected. Stars with a mass $\gtrsim 1.5 M_{\odot}$ typically contain hyperons in their core, and their presence is felt for the larger masses giving rise to a reduction of the star radius, and increasing uncertainties due to the largely unknown hyperon couplings.

Taking the whole set of models discussed in Sec. IV, we have confirmed the existence of a linear correlation between the symmetry energy slope and the radius. This correlation is stronger for the less massive stars when the central densities of the stars are below $2.5 n_{0}$, a conclusion first drawn in [89]. When larger masses are considered the spread of data increases, reflecting the lack of constraint to be imposed on the high density segment of the EOS. Considering the correlation between the incompressibility and the radius, the spread of data is independent of the mass of the star and prevents from extracting a clear correlation. These results imply that further tighter constraints on $L$ and $K$ are not expected to improve the radius uncertainty in an important way. 
A very promising avenue is given by the potential constraint imposed by the necessity of DUrca processes to operate in NS in order to explain the observations of thermal states of some of them as shown in [21]. Indeed, the restricted $L$ interval compatible with present terrestrial constraints is close to the threshold for DUrca process in nucleonic stars. This means that only a limited number of functionals can at the same time fulfill the $L$ constraint and allow DUrca processes in NS. However, it was also shown that if hyperons are included, the nucleonic DUrca process is shifted to lower densities, possibly allowing us to reconcile an efficient DUrca process and an $L$ restricted to the interval allowed by terrestrial experiments. This effect could be an indication of the presence of hyperons in the interior of a neutron star. A further constraint might be obtained if the mass of a NS with a low luminosity is measured.

Having shown the importance of using a unified EOS, all the studied EOS are accessible in the Supplemental Material and via the CompOSE database [20].

\section{ACKNOWLEDGMENTS}

The authors thank Isaac Vidaña, Stefan Typel, and Nicolas Chamel for their comments, Micaela Oertel for helpful discussions and assistance with the CompOSE database, and Helena Pais for supplying some RMF inner crust EOS. This work has been partially supported by New-Compstar COST MP1304 and by the NCN (Poland) Grant No. 2014/13/B/ST9/02621.

\section{APPENDIX: LINEAR MATCHING}

Let us assume that we want to perform the matching between an EOS for the core and one for the crust in the region defined by $P_{1}, \rho_{1}, \mu_{1}=\left(P_{1}+\rho_{1}\right) / n_{1}$ (highest pressure in the crust) and $P_{2}, \rho_{2}, \mu_{2}=\left(P_{2}+\rho_{2}\right) / n_{2}$ (lowest pressure in the core). For a linear matching of the form $P=a(\rho-\tilde{\rho})$ the dependence $P(\mu)$ reads

$$
P=P_{1}+\Delta P \frac{\mu^{b}-\left(\mu_{1}\right)^{b}}{\left(\mu_{2}\right)^{b}-\left(\mu_{1}\right)^{b}}, \quad b=\frac{1+a}{a},
$$

where $\Delta P=P_{2}-P_{1}$. Equation (A1) does not guarantee that the function $P(\rho)$ is continuous at $P=P_{1}$ and $P=P_{2}$. In general one has density jumps at these points corresponding to first-order phase transitions (see Fig. 4):

$$
\begin{gathered}
P=P_{1}: \quad n_{1} \rightarrow n_{1}^{\prime}, \quad \rho_{1} \rightarrow \rho_{1}^{\prime}, \\
P=P_{2}: \quad n_{2}^{\prime} \rightarrow n_{2}, \quad \rho_{2}^{\prime} \rightarrow \rho_{2}, \\
P_{1}<P<P_{2}, \quad \rho_{1}^{\prime}<\rho<\rho_{2}^{\prime}, \quad P=a(\rho-\tilde{\rho}) .
\end{gathered}
$$

TABLE VI. Numerical values for the matching presented in Fig. 4.

\begin{tabular}{cccccc}
\hline \hline$\mu_{2} / \mu_{1}$ & $b_{1}$ & $b_{2}$ & $a_{1}$ & $a_{2}$ & $\Delta P / \Delta \rho$ \\
\hline 1.0476 & 11.953 & 13.0004 & 0.0913 & 0.0833 & 0.088 \\
\hline \hline
\end{tabular}

From the continuity of $\mu$ at $P_{1}$ and $P_{2}$ we get

$$
\frac{b P_{2}+\tilde{\rho}}{b P_{1}+\tilde{\rho}}=q, \quad q \equiv\left(\frac{\mu_{2}}{\mu_{1}}\right)^{b} .
$$

Finally $\tilde{\rho}$ is given by

$$
\tilde{\rho}=b \frac{P_{2}-q P_{1}}{q-1} .
$$

The densities $\rho_{1}^{\prime}, \rho_{2}^{\prime}$ for a linear matching at $P_{1}$ and $P_{2}$ are given by the equations

$$
\begin{aligned}
& \rho_{1}^{\prime}=\tilde{\rho}+P_{1} / a=\frac{b}{q-1} \Delta P-P_{1}, \\
& \rho_{2}^{\prime}=\tilde{\rho}+P_{2} / a=\rho_{1}^{\prime}+\Delta P / a=\frac{b q}{q-1} \Delta P-P_{2} .
\end{aligned}
$$

The necessary conditions for the Rayleigh-Taylor stability in a star with a phase transition are $\rho_{1}^{\prime} \geqslant \rho_{1}$ and $\rho_{2}^{\prime} \leqslant \rho_{2}$, i.e.,

$$
\begin{aligned}
& \frac{b}{q-1} \Delta P \geqslant P_{1}+\rho_{1}=n_{1} \mu_{1}, \quad \frac{b}{q-1} \geqslant \frac{n_{1} \mu_{1}}{\Delta P}, \\
& \frac{b q}{q-1} \Delta P \leqslant P_{2}+\rho_{2}=n_{2} \mu_{2}, \quad \frac{b q}{q-1} \leqslant \frac{n_{2} \mu_{2}}{\Delta P} .
\end{aligned}
$$

Defining the function

$$
f(x)=\frac{x}{\left(\mu_{2} / \mu_{1}\right)^{x}-1},
$$

we get

$$
f(b) \geqslant \frac{n_{1} \mu_{1}}{\Delta P}, \quad f(-b) \leqslant \frac{n_{2} \mu_{2}}{\Delta P} .
$$

The equalities in Eq. (A12) correspond to the disappearance of the density jump at $P_{1}$ and $P_{2}$ respectively. The two solutions $\left(b_{1}, b_{2}\right)$ of the equations

$$
f\left(b_{1}\right)=\frac{n_{1} \mu_{1}}{\Delta P}, \quad f\left(-b_{2}\right)=\frac{n_{2} \mu_{2}}{\Delta P}
$$

define the maximum value of $b$ and the minimum of $a=$ $1 /(b-1)$ for which a linear matching is possible:

$$
b<b_{\max } \equiv \min \left(b_{1}, b_{2}\right), \quad a>a_{\min } \equiv \max \left(a_{1}, a_{2}\right) .
$$

Table VI gives numerical values for the example discussed in the text and presented in Fig. 4 with $\Delta \rho \equiv \rho_{2}-\rho_{1}$.
[1] P. B. Demorest, T. Pennucci, S. M. Ransom, M. S. E. Roberts, and J. W. T. Hessels, Nature (London) 467, 1081 (2010).

[2] E. Fonseca, T. T. Pennucci, J. A. Ellis et al., arXiv:1603.00545.

[3] J. Antoniadis et al., Science 340, 1233232 (2013).
[4] A. Y. Potekhin, Phys. Usp. 57, 735 (2014).

[5] A. W. Steiner, J. M. Lattimer, and E. F. Brown, Eur. Phys. J. A 52, 18 (2016).

[6] M. Fortin, J. L. Zdunik, P. Haensel, and M. Bejger, Astron. Astrophys. 576, A68 (2015). 
[7] W.-C. Chen and J. Piekarewicz, Phys. Rev. Lett. 115, 161101 (2015).

[8] K. C. Gendreau, Z. Arzoumanian, and T. Okajima, Proc. SPIE 8443, 844313 (2012).

[9] C. Motch, J. Wilms, D. Barret et al., arXiv:1306.2334.

[10] M. Feroci, J. W. den Herder, E. Bozzo et al., Proc. SPIE 8443, 84432D (2012).

[11] N. K. Glendenning, Compact Stars: Nuclear Physics, Particle Physics, and General Relativity (Springer, New York, 2000).

[12] G. Baym, C. Pethick, and P. Sutherland, Astrophys. J. 170, 299 (1971).

[13] J. S. Read, B. D. Lackey, B. J. Owen, and J. L. Friedman, Phys. Rev. D 79, 124032 (2009).

[14] F. Douchin and P. Haensel, Astron. Astrophys. 380, 151 (2001).

[15] E. Chabanat, P. Bonche, P. Haensel, J. Meyer, and R. Schaeffer, Nucl. Phys. A 635, 231 (1998).

[16] J. W. Negele and D. Vautherin, Nucl. Phys. A 207, 298 (1973).

[17] J. Piekarewicz, F. J. Fattoyev, and C. J. Horowitz, Phys. Rev. C 90, 015803 (2014).

[18] H.-J. Schulze and T. Rijken, Phys. Rev. C 84, 035801 (2011).

[19] D. Lonardoni, A. Lovato, S. Gandolfi, and F. Pederiva, Phys. Rev. Lett. 114, 092301 (2015).

[20] See Supplemental Material at http://link.aps.org/supplemental/ 10.1103/PhysRevC.94.035804 for tables of the EOS used in this work.

[21] M. V. Beznogov and D. G. Yakovlev, Mon. Not. R. Astron. Soc. 447, 1598 (2015).

[22] J. R. Oppenheimer and G. M. Volkoff, Phys. Rev. 55, 374 (1939); R. C. Tolman, ibid. 55, 364 (1939).

[23] P. Haensel and B. Pichon, Astron. Astrophys. 283, 313 (1994).

[24] S. B. Rüster, M. Hempel, and J. Schaffner-Bielich, Phys. Rev. C 73, 035804 (2006).

[25] N. Chamel, J. M. Pearson, A. F. Fantina et al., Acta Phys. Pol. B 46, 349 (2015).

[26] D. G. Ravenhall, C. J. Pethick, and J. R. Wilson, Phys. Rev. Lett. 50, 2066 (1983).

[27] C. J. Horowitz, M. A. Pérez-García, and J. Piekarewicz, Phys. Rev. C 69, 045804 (2004).

[28] C. J. Horowitz, M. A. Pérez-García, D. K. Berry, and J. Piekarewicz, Phys. Rev. C 72, 035801 (2005).

[29] T. Maruyama, T. Tatsumi, D. N. Voskresensky, T. Tanigawa, and S. Chiba, Phys. Rev. C 72, 015802 (2005).

[30] H. Sonoda, G. Watanabe, K. Sato, K. Yasuoka, and T. Ebisuzaki, Phys. Rev. C 77, 035806 (2008).

[31] G. Watanabe, H. Sonoda, T. Maruyama, K. Sato, K. Yasuoka, and T. Ebisuzaki, Phys. Rev. Lett. 103, 121101 (2009).

[32] S. S. Avancini, L. Brito, J. R. Marinelli, D. P. Menezes, M. M. W. de Moraes, C. Providência, and A. M. Santos, Phys. Rev. C 79, 035804 (2009); S. S. Avancini, S. Chiacchiera, D. P. Menezes, and C. Providência, ibid. 82, 055807 (2010); 85, 059904(E) (2012).

[33] S. S. Avancini, D. P. Menezes, M. D. Alloy, J. R. Marinelli, M. M. W. Moraes, and C. Providência, Phys. Rev. C 78, 015802 (2008).

[34] W. G. Newton and J. R. Stone, Phys. Rev. C 79, 055801 (2009).

[35] N. Yasutake, R. Łastowiecki, S. Benić, D. Blaschke, T. Maruyama, and T. Tatsumi, Phys. Rev. C 89, 065803 (2014).

[36] G. Baym, H. A. Bethe, and C. J. Pethick, Nucl. Phys. A 175, 225 (1971).

[37] N. K. Glendenning and S. A. Moszkowski, Phys. Rev. Lett. 67, 2414 (1991).
[38] F. Grill, H. Pais, C. Providência, I. Vidaña, and S. S. Avancini, Phys. Rev. C 90, 045803 (2014).

[39] G. A. Lalazissis, J. König, and P. Ring, Phys. Rev. C 55, 540 (1997).

[40] C. J. Horowitz and J. Piekarewicz, Phys. Rev. Lett. 86, 5647 (2001).

[41] P. S. Shternin, D. G. Yakovlev, P. Haensel, and A. Y. Potekhin, Mon Not. R. Astron Soc. 382, L43 (2007).

[42] D. Page and S. Reddy, Phys. Rev. Lett. 111, 241102 (2013).

[43] N. Andersson, K. Glampedakis, W. C. G. Ho, and C. M. Espinoza, Phys. Rev. Lett. 109, 241103 (2012).

[44] N. Chamel, Phys. Rev. Lett. 110, 011101 (2013).

[45] M. Gearheart, W. G. Newton, J. Hooker, and B.-A. Li, Mon. Not. R. Astron Soc. 418, 2343 (2011).

[46] Y. Sugahara and H. Toki, Nucl. Phys. A 579, 557 (1994).

[47] T. Gaitanos, M. Di Toro, S. Typel et al., Nucl. Phys. A 732, 24 (2004).

[48] S. K. Dhiman, R. Kumar, and B. K. Agrawal, Phys. Rev. C 76, 045801 (2007).

[49] B. K. Agrawal, Phys. Rev. C 81, 034323 (2010).

[50] G. A. Lalazissis, T. Nikšić, D. Vretenar, and P. Ring, Phys. Rev. C 71, 024312 (2005).

[51] S. Banik, M. Hempel, and D. Bandyopadhyay, Astrophys. J. Suppl. Ser. 214, 22 (2014).

[52] H. Shen, H. Toki, K. Oyamatsu, and K. Sumiyoshi, Nucl. Phys. A 637, 435 (1998).

[53] H. Shen, H. Toki, K. Oyamatsu, and K. Sumiyoshi, Prog. Theor. Phys. 100, 1013 (1998).

[54] S. Typel, G. Röpke, T. Klähn, D. Blaschke, and H. H. Wolter, Phys. Rev. C 81, 015803 (2010).

[55] F. Grill, C. Providência, and S. S. Avancini, Phys. Rev. C 85, 055808 (2012).

[56] T. Miyatsu, M.-K. Cheoun, and K. Saito, Phys. Rev. C 88, 015802 (2013).

[57] A. Sulaksono and B. K. Agrawal, Nucl. Phys. A 895, 44 (2012).

[58] I. Bednarek, P. Haensel, J. L. Zdunik, M. Bejger, and R. Mańka, Astron. Astrophys. 543, A157 (2012).

[59] F. Hofmann, C. M. Keil, and H. Lenske, Phys. Rev. C 64, 025804 (2001).

[60] J. Schaffner-Bielich, and A. Gal, Phys. Rev. C 62, 034311 (2000).

[61] M. Oertel, C. Providência, F. Gulminelli, and A. R. Raduta, J. Phys. G 42, 075202 (2015).

[62] H. Takahashi, J. K. Ahn, H. Akikawa et al., Phys. Rev. Lett. 87, 212502 (2001).

[63] I. Vidaña, A. Ramos, and A. Polls, Phys. Rev. C 70, 024306 (2004).

[64] K. Oyamatsu and K. Iida, Phys. Rev. C 75, 015801 (2007).

[65] E. Khan, J. Margueron, F. Gulminelli, and A. R. Raduta, Phys. Rev. C 92, 044313 (2015).

[66] P. Danielewicz and J. Lee, Nucl. Phys. A 818, 36 (2009).

[67] S. Goriely, N. Chamel, and J. M. Pearson, Phys. Rev. C 88, 024308 (2013).

[68] W. D. Myers and W. J. Swiatecki, Nucl. Phys. A 336, 267 (1980).

[69] P. Papakonstantinou, J. Margueron, F. Gulminelli, and Ad. R. Raduta, Phys. Rev. C 88, 045805 (2013).

[70] A. R. Raduta, F. Gulminelli, and F. Aymard, Eur. Phys. J. A 50, 24 (2014).

[71] F. Douchin and P. Haensel, Phys. Lett. B 485, 107 (2000). 
[72] F. Aymard, F. Gulminelli, and J. Margueron, Phys. Rev. C 89, 065807 (2014).

[73] H. S. Kohler, Nucl. Phys. A 258, 301 (1976).

[74] P.-G. Reinhard and H. Flocard, Nucl. Phys. A 584, 467 (1995).

[75] W. Nazarewicz, J. Dobaczewski, T. R. Werner, J. A. Maruhn, P. G. Reinhard, K. Rutz, C. R. Chinn, A. S. Umar, and M. R. Strayer, Phys. Rev. C 53, 740 (1996).

[76] E. Chabanat, Ph.D. thesis, University Claude Bernard Lyon-1, Lyon, France, 1995.

[77] E. Chabanat et al., Nucl. Phys. A 627, 710 (1997).

[78] L. Bennour, P. H. Heenen, P. Bonche, J. Dobaczewski, and H. Flocard, Phys. Rev. C 40, 2834 (1989).

[79] P. G. Reinhard, D. J. Dean, W. Nazarewicz, J. Dobaczewski, J. A. Maruhn, and M. R. Strayer, Phys. Rev. C 60, 014316 (1999).

[80] B. K. Agrawal, S. Shlomo, and V. K. Au, Phys. Rev. C 72, 014310 (2005).

[81] B. K. Agrawal, S. Shlomo, and V. Kim Au, Phys. Rev. C 68, 031304 (2003).

[82] J. Friedrich and P.-G. Reinhard, Phys. Rev. C 33, 335 (1986).

[83] S. Goriely, N. Chamel, and J. M. Pearson, Phys. Rev. C 82, 035804 (2010).

[84] A. F. Fantina, N. Chamel, J. M. Pearson, and S. Goriely, Astron. Astrophys. 559, A128 (2013).

[85] J. M. Pearson, N. Chamel, A. F. Fantina, and S. Goriely, Eur. Phys. J. A 50, 43 (2014).

[86] J. M. Pearson, S. Goriely and N. Chamel, Phys. Rev. C 83, 065810 (2011).

[87] J. M. Pearson, N. Chamel, S. Goriely, and C. Ducoin, Phys. Rev. C 85, 065803 (2012).

[88] J. M. Lattimer and M. Prakash, Phys. Rep. 442, 109 (2007).

[89] J. Carriere, C. J. Horowitz, and J. Piekarewicz, Astrophys. J. 593, 463 (2003).

[90] B. A. Brown, Phys. Rev. Lett. 85, 5296 (2000); S. Typel and B. A. Brown, Phys. Rev. C 64, 027302 (2001); A. W. Steiner, M. Prakash, J. Lattimer, and P. J. Ellis, Phys. Rep. 411, 325 (2005).

[91] M. Centelles, X. Roca-Maza, X. Vinas, and M. Warda, Phys. Rev. Lett. 102, 122502 (2009).

[92] J. Margueron, NewCompStar School 2016, http://compstar15. nipne.ro/.

[93] K. Hebeler, J. M. Lattimer, C. J. Pethick, and A. Schwenk, Astrophys. J. 773, 11 (2013).
[94] S. Gandolfi, J. Carlson, and S. Reddy, Phys. Rev. C 85, 032801 (2012).

[95] M. B. Tsang, J. R. Stone, F. Camera et al., Phys. Rev. C 86, 015803 (2012).

[96] J. M. Lattimer and Y. Lim, Astrophys. J. 771, 51 (2013).

[97] J. M. Lattimer and A. W. Steiner, Eur. Phys J. A 50, 40 (2014).

[98] M. Dutra, O. Lourenço, S. S. Avancini et al., Phys. Rev. C 90, 055203 (2014).

[99] J. Treiner, H. Krivine, O. Bohigas, and J. Martorell, Nucl. Phys. A 371, 253 (1981).

[100] J. M. Pearson, Phys. Lett. B 271, 12 (1991).

[101] J. R. Stone, N. J. Stone, and S. A. Moszkowski, Phys. Rev. C 89, 044316 (2014).

[102] S. Shlomo, V. M. Kolomietz, and G. Colo, Eur. Phys. J. A 30, 23 (2006).

[103] E. Khan, J. Margueron, and I. Vidana, Phys. Rev. Lett. 109, 092501 (2012).

[104] L.-W. Chen, C. M. Ko, B.-A. Li, and J. Xu, Phys. Rev. C 82, 024321 (2010).

[105] M. B. Tsang, Y. Zhang, P. Danielewicz, M. Famiano, Z. Li, W. G. Lynch, and A. W. Steiner, Phys. Rev. Lett. 102, 122701 (2009).

[106] X. Roca-Maza, M. Brenna, G. Colò et al., Phys. Rev. C 88, 024316 (2013).

[107] L. Trippa, G. Colò, and E. Vigezzi, Phys. Rev. C 77, 061304 (2008).

[108] M. Kortelainen, T. Lesinski, J. Moré et al., Phys. Rev. C 82, 024313 (2010).

[109] P. Danielewicz and J. Lee, Nucl. Phys. A 922, 1 (2014).

[110] E. Khan and J. Margueron, Phys. Rev. C 88, 034319 (2013).

[111] P. Haensel, A. Y. Potekhin, and D. G. Yakovlev, Neutron Stars 1. Equation of State and Structure (Springer, New York, 2007).

[112] J. M. Lattimer, C. J. Pethick, M. Prakash, and P. Haensel, Phys. Rev. Lett. 66, 2701 (1991).

[113] T. Klahn, D. Blaschke, S. Typel, E. N. E. vanDalen, A. Faessler, C. Fuchs, T. Gaitanos, H. Grigorian, A. Ho, E. E. Kolomeitsev, M. C. Miller, G. Ropke, J. Trumper, D. N. Voskresensky, F. Weber, and H. H. Wolter, Phys. Rev. C 74, 035802 (2006).

[114] S. Popov, H. Grigorian, R. Turolla, and D. Blaschke, Astron. Astrophys. 448, 327 (2006).

[115] M. Prakash, M. Prakash, J. M. Lattimer, and C. J. Pethick, Astrophys. J. 390, L77 (1992). 